\title{
Toward an Understanding of the Structural and Mechanistic Aspects of Protein-Protein Interactions in 2-Oxoacid Dehydrogenase Complexes
}

\author{
Natalia S. Nemeria ${ }^{1, *}$, Xu Zhang ${ }^{1, *} \mathbb{D}$, Joao Leandro ${ }^{2}$, Jieyu Zhou ${ }^{1}$, Luying Yang ${ }^{1}$, Sander M. Houten ${ }^{2}$ and \\ Frank Jordan $1, * \mathbb{D}$ \\ 1 Department of Chemistry, Rutgers, The State University of New Jersey, Newark, NJ 07102, USA; \\ jieyu.zhou@abzena.com (J.Z.); yly19911008@gmail.com (L.Y.) \\ 2 Department of Genetics and Genomic Sciences, Icahn School of Medicine at Mount Sinai, \\ New York, NY 10029, USA; joao.leandro@mssm.edu (J.L.); sander.houten@mssm.edu (S.M.H.) \\ * Correspondence: nemeria@newark.rutgers.edu (N.S.N.); xz364@iqb.rutgers.edu (X.Z.); \\ frjordan@newark.rutgers.edu (F.J.)
}

check for updates

Citation: Nemeria, N.S.; Zhang, X.; Leandro, J.; Zhou, J.; Yang, L.; Houten, S.M.; Jordan, F. Toward an Understanding of the Structural and Mechanistic Aspects of Protein-Protein Interactions in 2-Oxoacid Dehydrogenase Complexes. Life 2021, 11, 407. https://doi.org/10.3390/ life11050407

Academic Editor: Sonia Longhi

Received: 3 April 2021

Accepted: 22 April 2021

Published: 29 April 2021

Publisher's Note: MDPI stays neutral with regard to jurisdictional claims in published maps and institutional affiliations.

Copyright: (c) 2021 by the authors. Licensee MDPI, Basel, Switzerland. This article is an open access article distributed under the terms and conditions of the Creative Commons Attribution (CC BY) license (https:/ / creativecommons.org/licenses/by/ $4.0 /)$.
Abstract: The 2-oxoglutarate dehydrogenase complex (OGDHc) is a key enzyme in the tricarboxylic acid (TCA) cycle and represents one of the major regulators of mitochondrial metabolism through NADH and reactive oxygen species levels. The OGDHc impacts cell metabolic and cell signaling pathways through the coupling of 2-oxoglutarate metabolism to gene transcription related to tumor cell proliferation and aging. DHTKD1 is a gene encoding 2-oxoadipate dehydrogenase (E1a), which functions in the L-lysine degradation pathway. The potentially damaging variants in DHTKD1 have been associated to the (neuro) pathogenesis of several diseases. Evidence was obtained for the formation of a hybrid complex between the OGDHc and E1a, suggesting a potential cross talk between the two metabolic pathways and raising fundamental questions about their assembly. Here we reviewed the recent findings and advances in understanding of protein-protein interactions in OGDHc and 2-oxoadipate dehydrogenase complex (OADHc), an understanding that will create a scaffold to help design approaches to mitigate the effects of diseases associated with dysfunction of the TCA cycle or lysine degradation. A combination of biochemical, biophysical and structural approaches such as chemical cross-linking MS and cryo-EM appears particularly promising to provide vital information for the assembly of 2-oxoacid dehydrogenase complexes, their function and regulation.

Keywords: neurodegeneration; glucose metabolism; enzyme catalysis; protein-protein interaction; hydrogen exchange mass spectrometry; protein cross-linking; protein assembly; molecular modeling

\section{Introduction}

The human 2-oxoglutarate dehydrogenase complex (OGDHc) is a key enzyme in the tricarboxylic acid (TCA) cycle, which is a common pathway for oxidation of fuel molecules, including carbohydrates, fatty acids, and amino acids. Diminished OGDHc activity and mitochondrial abnormalities have been correlated with numerous neurodegenerative disorders, including Alzheimer's disease, however, a link between reductions in the mitochondrial TCA cycle enzymes and (neuro) degeneration has not been established so far [1-4]. Recently, a biallelic pathogenic variant in OGDH gene leading to deficient 2-oxoglutarate dehydrogenase (E1o, the first component of the OGDHc, also known as $\mathrm{OGDH}$ ) was reported in individuals with a neurological disorder resembling mitochondrial disease [5]. While rare in $O G D H$, whole-exome sequencing and rare variant burden analysis determined an overabundance of putative, potentially damaging mutations in the OGDHL (OGDH-like) and DHTKD1 genes across multiple patients with eosinophilic esophagitis (EoE), a chronic allergic disorder that presents in infancy and in adulthood [6]. The OGDHL encodes a putative 2-oxoglutarate dehydrogenase-like protein (E1o-like) in the TCA cycle that 
is tissue-specific and is mainly expressed in brain and liver (Scheme 1) [7]. Furthermore, a homozygous deleterious variant (c.2333C > T; p. Ser778Leu) was recently identified in OGDHL and was associated with neuro-degenerative phenotype in patients [8]. The DHTKD1 gene encodes a less-known homologue of E1o, the enzyme 2-oxoadipate dehydrogenase (E1a, also known as DHTKD1) in the L-lysine degradation pathway (Scheme 1). Genetic studies have linked variants in DHTKD1 to the (neuro) pathogenesis of several metabolic disorders: $\alpha$-aminoadipic and $\alpha$-ketoadipic aciduria (AMOXAD: MIM 204750) [9-11], CharcotMarie-Tooth disease type 2Q (CMT2Q: MIM 615025) [12-14] and eosinophilic esophagitis (EoE), a chronic allergic disorder [6].

Pharmacological inhibition of E1a has been proposed as a strategy for substrate reduction therapy to treat glutaric aciduria type 1 (GA1: MIM 231670), a metabolic disorder that is caused by mutations in the $G C D H$ gene encoding the mitochondrial protein glutaryl-CoA dehydrogenase (GCDH; EC 1.3.8.6) located downstream of E1a in the L-lysine degradation pathway (Scheme 1) [15].

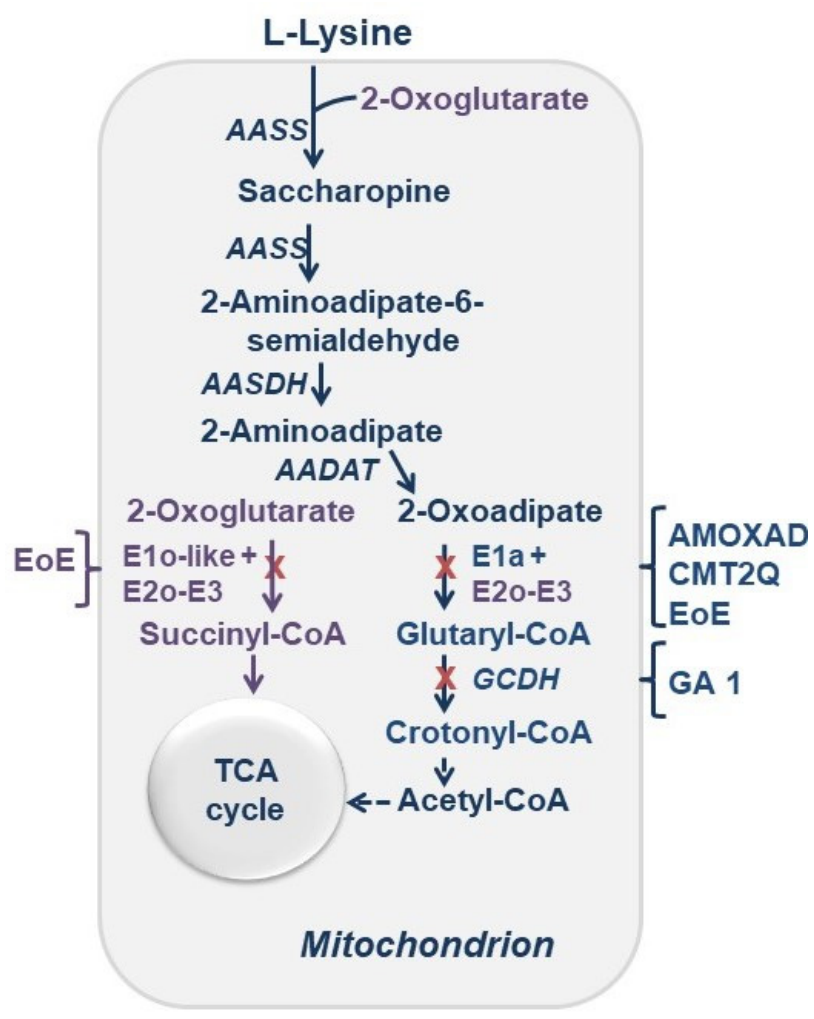

Scheme 1. Role of the OGDHc in the TCA cycle and of the E1a in the L-lysine degradation pathway in health and diseases. AASS, 2-aminoadipate-6-semialdehyde synthase; $A A S D H, 2$-amino-adipate-6semialdehyde dehydrogenase; $A A D A T$, 2-aminoadipate transaminase; $G C D H$, glutaryl-CoA dehydrogenase; E1o-like, E2o, E3-components of the OGDHc; E1a, 2-oxoadipate dehydrogenase; AMOXAD, $\alpha$-aminoadipic and $\alpha$-ketoadipic aciduria; CMT2Q, Charcot-Marie-Tooth disease type 2Q; EoE, eosinophilic esophagitis; GA1, glutaric aciduria 1.

Despite the reported genetic findings, no pathophysiologic mechanism has been reported for the disease-associated OGDH, OGDHL and DHTKD1 variants and their impact on E1o, E1o-like and E1a protein structure and function.

Diverse functions of the E1o have been shown to be associated with cancer. As the OGDHc is integrated within mitochondrial functions and represents one of the major regulators of mitochondrial metabolism, it was suggested that it is pivotal in the adaptation of cancer cells to a demanding environment in vivo [16]. Two mitochondrial genes $O G D H$ and LIAS (encoding lipoic acid synthase) were identified that are involved in the mechanism of regulation in hypoxia-inducible transcription factors (HIFs) under aerobic 
conditions [17]. Lu et al. suggested that OGDHc mediates SIRT5 (sirtuin 5, an NAD ${ }^{+}$-dependent protein deacylase) function, a potential suppressor of cell growth and migration in gastric cancer [18]. The direct interaction between SIRT5 and OGDHc was shown and it was reported that desuccinylation of OGDHc by SIRT5 inhibits OGDHc activity [18]. The authors suggested that SIRT5 suppressed gastric cancer cell growth and migration by inhibiting mitochondrial function and by increasing ROS production via down-regulation of OGDHc activity. Thus, both SIRT5 and OGDHc could be novel therapeutic targets for gastric cancer treatment $[18,19]$. Several recent studies support the rationale to target individual components of the OGDHc, specifically E1o [20] or E2o [21], or possibly all three components of the OGDHc [22] for cancer treatment [23].

Recent findings suggested that post-translational modifications (PTMs) by succinylation and glutarylation could be one of the mechanisms by which cells adapt to dynamic environmental changes to maintain metabolic homeostasis including regulation of energy production [24-28]. The significance of lysine succinylation and glutarylation has begun to be revealed for nuclear proteins, while its significance for cytosolic and mitochondrial proteins has been examined in only a few proteins $[25,29,30]$. Thus, glutarylation of the Lys91 in histone H4 in mammalian cells was reported [31]. According to a suggested mechanism, a known histone acetyltransferase KAT2A when coupled with the OADHc, acts as a histone glutaryltransferase in cells, while SIRT7 acts as an "eraser" of the glutarylated Lys91 at histone H4 [31]. On the flip side, when KAT2A is coupled with the OGDHc, it could recognize succinyl-CoA and transfer the succinyl group to the Lys79 of histone $\mathrm{H} 3$ [32]. These findings provided evidence for a tight link between metabolism and epigenetic regulation of gene expression by succinylation and glutarylation [31].

Earlier evidence suggested that OGDHc and its E2o component could function as a succinyltransferase for modification of cytosolic and mitochondrial proteins in cultured neurons and in neuronal cell lines and could provide an efficient mechanism to coordinate metabolic pathways at the cellular level $[3,27]$. Recent findings in vitro suggested that the E2o could also serve as a glutaryltransferase, in addition to functioning as a succinyltransferase, thus providing an efficient mechanism to coordinate the TCA cycle and the L-lysine metabolic pathway at the cellular level in normal state and in disease [33]. Increased lysine glutarylation of mitochondrial proteins in the brain and liver was observed in a GA1 mouse model $[25,30]$. However, the molecular mechanisms underlying the pathogenesis of GA1 are still poorly understood.

Finally, a novel function of E1o in host cell metabolic adaptation to viral infection was suggested [34]. It was found that in response to viral infection, host cells impair the activity of the $N^{6}$-methyladenosine $\left(\mathrm{m}^{6} \mathrm{~A}\right) \mathrm{RNA}$ demethylase leading to increased $\mathrm{m}^{6} \mathrm{~A}$ methylation on the E1o mRNA and to reduced mRNA stability. The reduced E1o protein expression led to reduced production of the itaconate intermediate in the TCA cycle, an intermediate which is required for viral replication, thus providing a crosstalk between $\mathrm{m}^{6} \mathrm{~A}$ RNA modification and metabolic reprogramming via the E1o-itaconate pathway [34]. The E1o-itaconate metabolic response was suggested as a potential therapeutic target for the control of viral infection [34]. In view of the accumulated data on the physiological importance of the OGDHc and OADHc in human health and disease, and their role as potential therapeutic targets, we first review OGDHc function and discuss a novel alternative mechanism of transthiolacylation catalysis in the active centers of the E. coli E2o that would be applicable to all E2 components. Next, we discuss protein-protein interactions identified in the human E1o-E2o, E1o-E3 and E3-E2o binary sub-complexes by hydrogen-deuterium exchange MS (HDX-MS) and chemical cross-linking MS (CL-MS), leading to the remarkable conclusion that the N-terminal region of E1o may constitute a unique dual-subunit-binding domain (DSBD) in human OGDHc, which is recognized by both the E2o and E3 components. Of special interest is recognition of the formation of a hybrid complex between OGDHc and OADHc. In the second half of this review, we discuss the structural insight into the architecture of the human E1a active site during catalysis, of which two independent X-ray structures have been reported recently $[35,36]$. 
Taking into consideration both in vitro and in vivo evidence for the interaction between E1a and E2o from two distinct metabolic pathways, we next present the models constructed for the E1o-E2o and E1a-E2o assembly to advance our understanding of protein-protein interactions in human OGDHc and OADHc in the absence of X-ray crystallographic or cryo-EM-based atomic structure of intact OGDHc or OADHc.

\section{The 2-Oxoglutarate Dehydrogenase Complex}

\subsection{Overview of the Molecular Mechanism of the OGDHc}

The canonical human OGDHc is a key enzyme in the TCA cycle, comprising multiple copies of three components, E1o, E2o, and E3. The five principal chemical steps involved in the reactions yielding succinyl-CoA are shown in Figure 1A. The ThDP-dependent E1o catalyzes the decarboxylation of 2-oxoglutarate (OG) $\left(k_{2}, k_{3}\right)$, followed by reductive succinylation of the E2o $\left(k_{4}, k_{5}\right)$. The reductive acylation is the important coupling mechanism of the reactions of the E1 and E2 components in 2-oxoacid dehydrogenase complexes, sometimes called substrate channeling. It is generally accepted that lipoyllysyl-E2 (lipoic acid is covalently amidated onto the $\varepsilon$-amino group of a lysine residue of E2 in its N-terminal region, the so called lipoyl domain or LDo) is the oxidizing agent for the enamine product of OG decarboxylation, which is non-covalently assembled with the E1 component [37]. Below we will focus on mechanism for the reductive acylation reaction between the E1 and E2o components and on the synthesis of the acyl-CoA in the active centers of E2o which is a controversial topic.

\subsubsection{Mechanism for the Reductive Acylation Reaction}

Over the years two mechanisms have been proposed for the reductive acylation reaction: (a) A stepwise mechanism, in which the redox process, i.e., electron transfer, the oxidation of the enamine to the acyl-ThDP with concomitant reduction of the lipoyllysyl-E2 to dihydrolipoyllysyl-E2 is the first step, followed by group transfer [38]; (b) A concerted mechanism, in which the enamine $\mathrm{C} 2 \alpha$-carbon as a nucleophile attacks the $S 8$ atom of the lipoyllysyl-E2, opening the dithiolane ring momentarily and forming a tetrahedral intermediate, thereby transiently linking the two components, the reaction being completed by cleavage of the $\mathrm{C} 2 \alpha-\mathrm{C} 2$ bond thus regenerating the ThDP ylide and generating the acyl-S8-dihydrolipoate (see Scheme 2 for a concerted mechanism of the OADHc with 2-oxoadipate) $[37,39]$. According to the second mechanism, electron and group transfer are synchronized, necessitating approach of the lipoyllysyl-E2 to within C-S bond distance to the $\mathrm{C} 2 \alpha$-carbon of the substrate in the first post-decarboxylation step. Yet, even according to the first mechanism, any plausible transfer of the acyl group to the S8-thiolate would require close approach of the lipoyllysyl group to the carbonyl group of the C2-acylThDP. A chemical model developed at Rutgers earlier was more consistent with the second mechanism, proceeding via a tetrahedral intermediate [39]. An appropriate model reaction to determine the rate of reductive acyl transfer from E1 to the lipoyllysyl-E2 has been developed (Figure 1C) [40-43], which maintains both the chemistry and the inter-component communication due to its specific recognition of E1 [44-46]. The full-length E2o is a highly segmented protein, comprising from the N-terminal end, a single lipoyl domain (LDo), linker region and an acyltransferase catalytic domain or E2o core domain (Figure 2A). By using the independently expressed LDo in place of intact E2o, the mass of the acylated and unacylated lipoyl domain could be measured by mass-spectrometry (FT-MS) with high precision, an experiment that at the same time enabled calculation of the rate constant $\left(k_{4}\right.$ in Figure 1A) for acyl transfer between the E1 and E2 components [43]. 
A

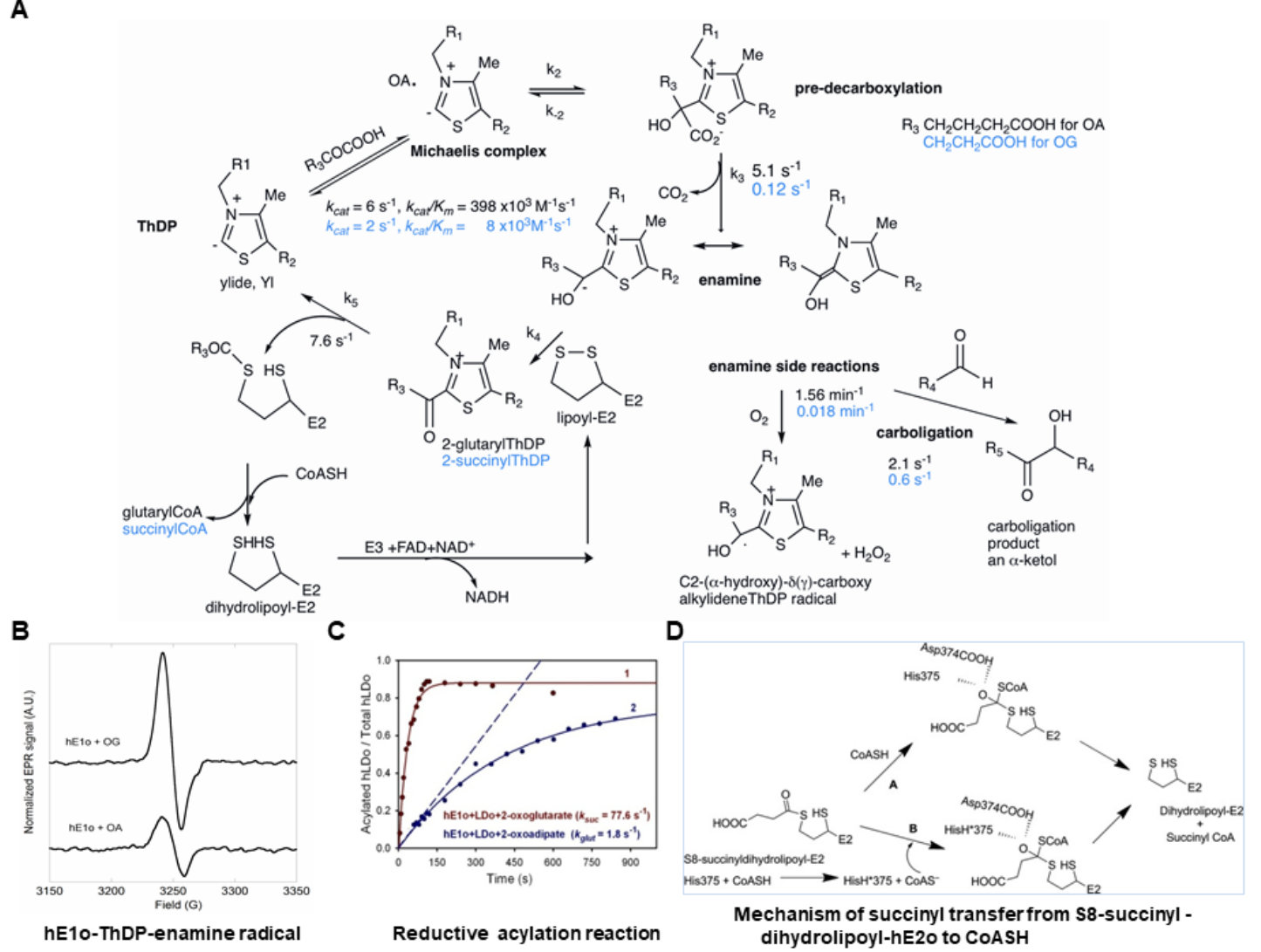

Figure 1. (A) Mechanism of the OGDHc with 2-oxoglutarate (in black) and 2-oxoadipate (in blue) as substrates. (B) X-band EPR spectra of the ThDP-enamine radical species generated on E1o OG and OA. The radical species generated on E1o from OA is $\sim 3$ times lower in concentration compared to that from OG $(0.9 \mu \mathrm{M}$ with $\sim 0.2 \%$ occupancy of E1o active centers). (C) Time dependence of reductive acylation of the lipoyllysyl groups on LDo by E1o from OG or OA could be analyzed by FT-MS. The fraction of reductively acylated LDo versus total LDo (acylated plus un-acylated LDo) when plotted versus time, allowed evaluation of the rate of acyl transfer from E1o to E2o $\left(k_{4}\right.$ in (A)). (D) Mechanism of succinyl transfer from S8-succinyldihydrolipoyllysyl-E2o to CoASH with suggested role of His375 and Asp374. Pathway A: direct attack by the conjugate base thiolate anion of CoASH assuming a low $\mathrm{pK}_{\mathrm{a}}$ for the $\mathrm{CoASH}$, or by the thiol form itself. Pathway B: initial proton transfer from CoASH to His375 forming the conjugate base $\mathrm{CoAS}^{-}$, which is the attacking agent. Both pathways then proceed by an oxyanionic tetrahedral intermediate [41].

\subsubsection{Synthesis of Succinyl-CoA}

The reductive succinylation reaction is followed by trans-thiolesterification of the succinyl group onto CoA in the active centers of hE2o (i.e., in the catalytic domain, CDo), generating succinyl-CoA (Figure 1A). The enzymatic mechanism responsible for synthesis of succinyl-CoA has been elucidated for the E. coli E2o (Figure 1D) [41], a mechanism that is applicable to all E2o components due to highly conserved structure of the reported E2o active centers [47-52]. There are two likely mechanisms to account for the transfer of an acyl group between two thiols in Figure 1D. A general acid-base mechanism would suggest that the catalytically important His375 of the E. coli E2o catalytic domain converts the thiol group of the attacking nucleophile $(\mathrm{CoASH})$ to a thiolate anion $\left(\mathrm{CoAS}^{-}\right)$as depicted on pathway B in Figure 1D. The activated thiolate then attacks the carbonyl atom of the succinyldihydrolipoyl-E2o to form a tetrahedral intermediate, which is stabilized by the hydroxyl side chain of Thr323 [48]. This mechanism was suggested based on analogy with a mechanism developed for chloramphenicol acetyltransferase [53-55]. However, the catalytic efficiency $\left(k_{c a t} / K_{m}\right)$ of the His375Ala substituted E. coli E2o was reduced by only 54-fold compared to unsubstituted E. coli E2o, while a $9 \times 10^{5}$-fold reduction in catalytic efficiency was determined for the analogous His195Ala substitution in chloramphenicol 
acetyltransferase, suggesting different functions for the highly conserved histidine residues on the two enzymes [41]. As an alternative to acid-base catalysis, a direct attack by a thiolate anion on the thiol ester carbon had been suggested, forming a symmetrical tetrahedral oxyanionic intermediate, in which the central carbon atom is flanked by two C-S bonds with nearly equal probability for cleavage [41]. Evidence was provided that His375 and Asp374 play a role in the stabilization of this symmetrical tetrahedral oxyanionic intermediate by formation of two hydrogen bonds, rather than in acid-base catalysis (pathway A in Figure 1D). An important conclusion from these studies is that succinyl transfer to CoA and release of succinyl-CoA, rather than the reductive succinylation reaction, is the ratelimiting step [41]. Next, the E3 re-oxidizes dihydrolipoyllysyl E2o with concomitant reduction of $\mathrm{NAD}^{+}$to $\mathrm{NADH}$ (Figure $1 \mathrm{~A}$ ). The E3 is shared by all members of the 2-oxo acid dehydrogenase complex family in mammalian cells.

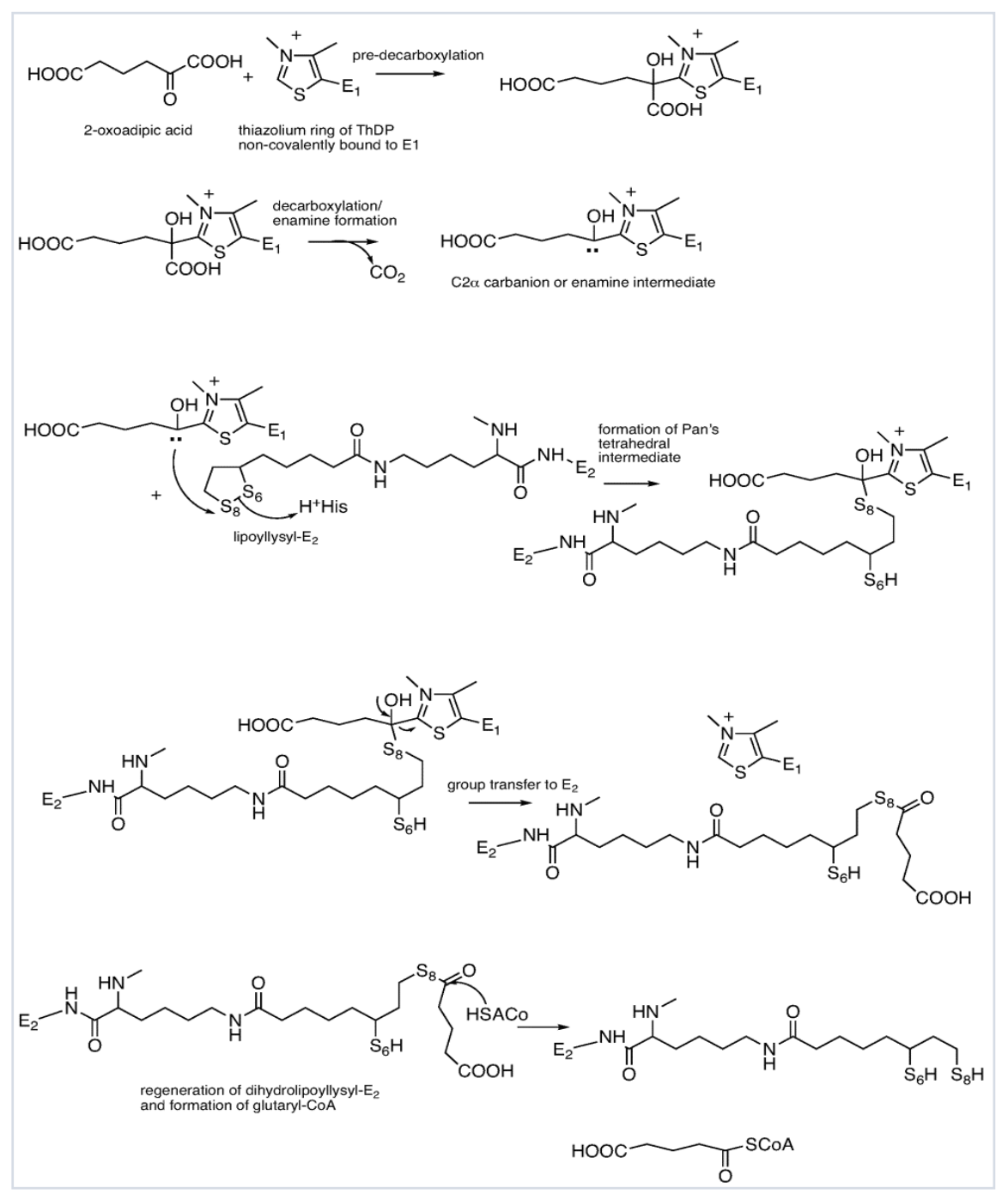

Scheme 2. Mechanism of the OADHc (comprising multiple copies of the E1a, E2o and E3 components) showing the formation of the enamine intermediate on E1a from 2-oxoadipate and reductive glutarylation reaction between E1o and E2o according to a concerted mechanism [39].

The human OGDHc is also recognized as a significant source of superoxide radical anion and $\mathrm{H}_{2} \mathrm{O}_{2}$ (reactive oxygen species, $\mathrm{ROS}$ ) that could lead to oxidative stress in mitochondria [56-64]. While earlier this function was assigned to the E3 component in the reverse direction $[56,57,59,61]$, formation of the ThDP-enamine radical species in the active centers of E1o from 2-oxoglutarate and 2-oxoadipate in the physiological direction was demonstrated by 
electron paramagnetic resonance spectroscopy and represents an "off-pathway" side reaction comprising less than 1\% of "on-pathway" reactivity (Figure 1B) $[43,60,63,64]$, as suggested earlier for the E. coli E1o [65].

\subsection{Protein-Protein Interactions in $\mathrm{OGDHc}$}

\subsubsection{Interactions in Binary E1o-E2o, E1o-E3 and E2o-E3 Subcomplexes}

A typical approach to define protein-protein interactions in multi-enzyme complexes, is to first determine the structure of the individual components. Currently, there is no X-ray structure of the human E1o. There are, however, X-ray structures available for the N-terminally truncated E. coli $\mathrm{E}^{1} \mathrm{o}_{\Delta 77}(39 \%$ identity, missing the N-terminal 77 amino acids) [66], the N-terminally truncated Mycobacterium smegmatis $\alpha$-ketoglutarate decarboxylase $\left(M s K G D_{\Delta 115}, 41 \%\right.$ identities, missing the $\mathrm{N}$-terminal 115 amino acids) [67], the $M_{s K G D}{ }_{\triangle 360}$ catalytic domain, which showed overall structural similarity to the $E$. coli E1o [68], and the $M s K_{G} D_{\Delta 360}$ catalytic domain in complex with phosphonate analogues of OG [69]. X-ray structures were recently reported for the $\mathrm{N}$-terminally truncated human E1a (residues 45-919, missing the N-terminal 44 residues) at $1.9 \AA$ [35] and for human E1a (residues 25-919, missing the N-terminal 24 residues) at $2.25 \AA$ resolution (38.5\% identities between E1o and E1a) [36]. Single-particle cryo-EM reconstruction of the E2o inner core domain at $4.7 \AA$ global resolution [35] has been reported showing 24 E2o catalytic domains assembled as eight trimers and positioned at each of the eight vertices of the cubic core with octahedral symmetry (Figure 2B). However, there is no atomic resolution structure reported for any of the 2-oxo acid dehydrogenase complexes. In the absence of the direct insight into assembly of the components into OGDHc, a multipronged approach has been employed including fluorescence, HDX-MS and CL-MS studies which allowed evaluation of the strength and loci of interaction in binary E1o-E2o, E1o-E3 and E2o-E3 sub-complexes [70]. Among the remarkable findings is that in the binary sub-complexes, strong interactions ( $K_{d}$ in the $0.04-0.14 \mu \mathrm{M}$ range) were demonstrated between the E1o and E2o components, but not between the E2o and E3. Importantly, two peptides from the $\mathrm{N}$-terminal region of E1o comprising residues ${ }^{18}$ YVEEM $^{22}$ and ${ }^{27}$ LENPKSVHKSWDIF $^{40}$ were identified that represent the most likely candidates for the interaction of E1o with both E2o and E3 [70]. The important role of the E2o region comprising residues from both the E2o core domain and the linker region was identified for the first time as critical for E1o-E2o interactions (Figure 2C,D) and represents a unique E2o-binding mode in human OGDHc [70]. In contrast, there was no evidence for E2o-E3 interaction indicating that there was only a transient interaction between these two components, too weak to be captured by the methods applied. These findings are in accord with the accepted OGDHc mechanism in Figure 1A, where with each turnover, the reduced lipoyl domain (dihydrolipoyllysilylgroup on E2o) must be re-oxidized by the FAD on E3 [71] In summary, the N-terminal region of E1o (residues 18-40) could constitute a unique dual-subunit-binding domain (DSBD) in human OGDHc, which is recognized by both the E2o and E3 components, suggesting that an initial formation of the uniquely strong E1o-E2o interaction could facilitate assembly with E3 into OGDHc, a hypothesis that needs to be confirmed in further studies. 
A

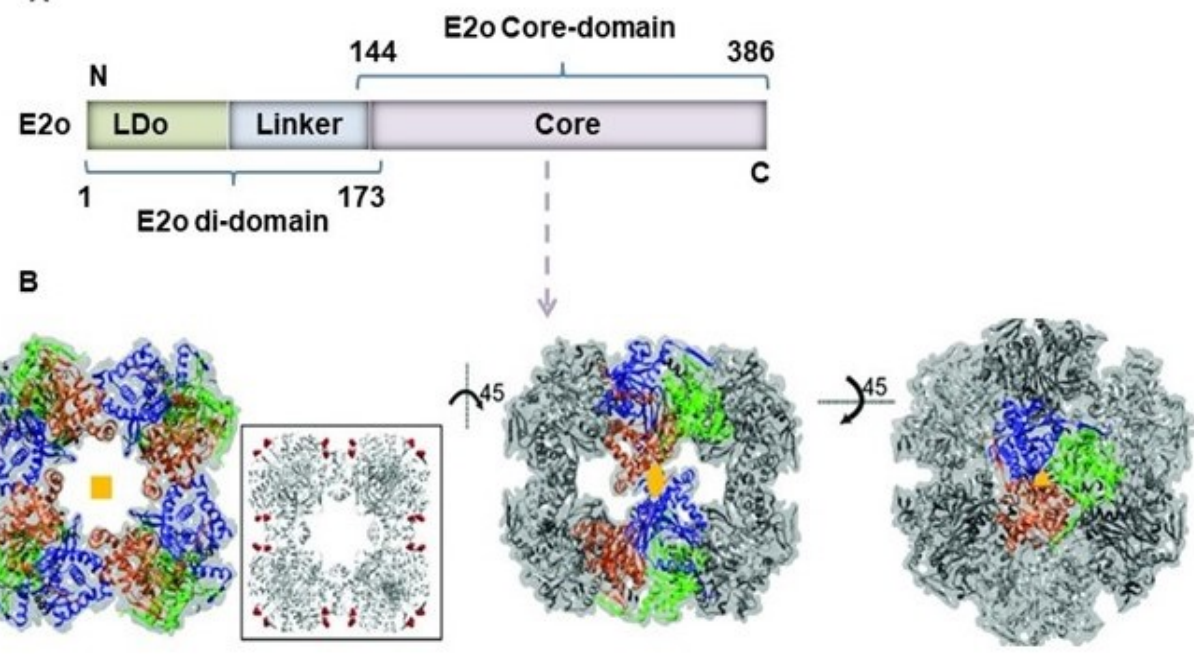

C

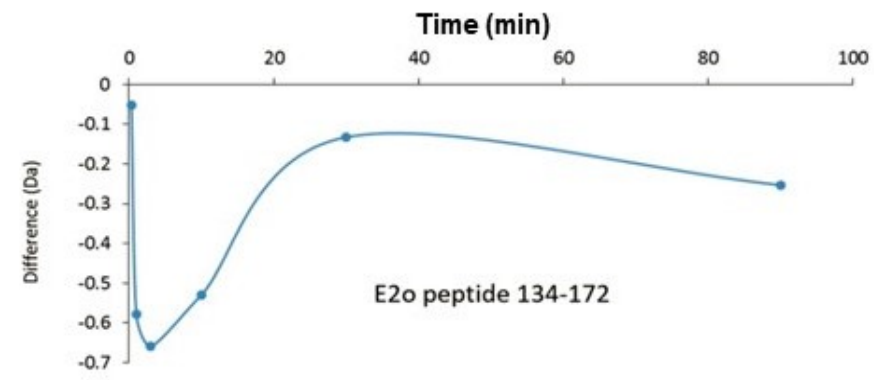

D

\begin{tabular}{|c|c|}
\hline $\mathrm{hE}_{20}$ & AEAGAGKGLRSEHREKMNRMRQRIAQRLKE \\
\hline$E C E_{2 p}$ & ATPLIRRLAREFGVNLAKVKGTGRKGRIL-REDVQAYVKEAI----- \\
\hline$E_{21}$ & TPAVRRLAMENNIKLSEVVGSGKDGRIL-KEDILNYLEKQT----- \\
\hline & VSPLAKKLAVVEKGIDLTQVKGTGPDGRIT-KKDIDSFV-------- \\
\hline & AMPSVRKYAREKGVDIRLVQGTGKNGRVL-KEDIDAFLAGGA---- \\
\hline pE & ASPAVRKRALDAGIELRYVHGSGPAGRIL-HEDLDAFMS---- \\
\hline & LSPAIRRLLAEHNLDASAIKGTGVGGRLT-REDVEKHLAKAP- \\
\hline & PAARKIAEENAIAADSITGTGKGGRVT-KEDAVAAAE---- \\
\hline
\end{tabular}

Figure 2. (A) Domain structure of the human E2o showing the lipoyl domain (LDo) and the E2o core or catalytic domain (CDo) connected by a linker region. (B) Electron micrograph reconstruction of the human E2o core domain structure at $4.7 \AA$ resolution [35]. Figure reproduced with permission of the International Union of Crystallography). The trimer building blocks (the individual subunits are shown in blue, green and orange) are assembled into the 24-meric core via four-fold (left), two-fold (middle) and three-fold symmetry axes, respectively. Insert shows the first residue 219 (red spheres) from each subunit of the 24-mer E2o core which is exposed to the surface of the E2o core. (C) On human E2o, the region comprising residues from the core domain (in bold) and E2o linker region ( ${ }^{144}$ AEAGAGKGLRSEHREKMNRMRQRIAQRLKE ${ }^{174}$ ) displayed a significant decrease in the level of the deuterium uptake during the first 3 min upon E1o binding, suggesting a unique subunit-binding mode in human OGDHc assembly, where the E2o core domain also participates in the interaction with E1o. (D) Sequence alignment of the human E2o linker-core region involved in interaction with E1o with some known E1-binding domains in E2 components identified conserved residues, indicating that E2o shares some but not all sequence features of other subunit-binding domains involved in E1o binding. The abbreviations are denoted: human (h), Escherichia coli (Ec), Bacillus stearothermophilus (Bst), Pseudomonas putida (Pp), and Azotobacter vinelandii (Av), 2-oxoglutarate dehydrogenase complex (o), pyruvate dehydrogenase complex (p), and branched chain 2-oxoacid dehydrogenase complex (b). Alignment of multiple sequences was carried out using the Clustal Omega program with default settings [70].

\subsubsection{The Assembly of E3 into OGDHc}

Related to the assembly of E3 into OGDHc, a novel structural component of the mitochondrial OGDHc named as $\mathrm{Kgd} 4$ (Ymr31) was identified in yeast, previously described as a part of the mitochondrial ribosome with a role in recruiting the E3 subunits to OGDHc [72]. A Kgd4 homologue was also identified as part of the murine OGDHc (MRPS36) [72]. A model was suggested for organization of the mitochondrial OGDHc where Kgd4 binds to 
the preformed E1-E2 core to recruit the E3 subunit into the complex while in the absence of $\mathrm{Kgd} 4$ the binding of $\mathrm{E} 3$ to the core is dramatically reduced [72]. However, in recently reported studies by Houten's group, the transient transfection of MRPS36 encoded protein together with the E1o (E1a), E2o and E3, did not increase the activity of the OGDHc or of OADHc in HEK-293 cell lysates with OG and OA as substrates [73], indicating a different assembly of the E3 in these human complexes. Notably, the human E3 which is shared by all members of the 2-oxoacid dehydrogenase complex family in mammalian cells has a different binding mode in the human pyruvate dehydrogenase complex (PDHc), where the pyruvate dehydrogenase component (E1p) binds to the E1p-binding domain on E2p (peripheral subunit-binding domain, PSBD), while the E3 binds to the E3-binding protein (E3BP, formerly known as Protein X), a component with no known catalytic function [74]. The E2p and E3BP (typically co-expressed) form the inner core of the mammalian PDHc according to two distinct "substitution" models proposed: 48 subunits of E2p + 12 subunits of E3BP or 40 subunits of E2p + 20 subunits of E3BP in the 60-meric inner core [75-80]. Structure determination of the human E2p inner core at $3.1 \AA$ atomic resolution [79], and of the full-length human E2p-E3BP assembly at $6.3 \AA$ resolution [80] have been reported recently by using cryo-EM and single particle reconstruction (see below). More questions have been raised and need to be answered regarding the E1o-E2o interaction in human OGDHc since the recent finding that the human 2-oxoadipate dehydrogenase (E1a) of the L-lysine, L-hydroxylysine and L-tryptophan catabolic pathway and the E1o of the OGDHc in the TCA cycle share the same E2o component and can utilize the recycling universally used E3 component for their function, while earlier the formation of hybrid complexes between OGDHc and PDHc had been reported.

\subsection{The Newly Revealed Feature of the OGDHc in the TCA Cycle, the Propensity to Form a Hybrid Complex with E1a Ftom the L-Lysine Degradation Pathway}

The first evidence for the formation of a hybrid 2-oxo acid dehydrogenase complex involving the E1o, E2o and E3 components of the OGDHc and the E1p component of the PDHc had been reported more than three decades ago for E. coli cells, with no physiological importance attributed to such a hybrid complex [81]. Later, the unique properties with respect to their protein components and three-dimensional organization of the PDHc and OGDHc from Gram-positive bacteria such as Mycobacterium tuberculosis (Mtb) and Corynebacterium glutamicum were reported $[82,83]$. There is an E2p component in these bacteria, which on assembly with E1p and E3 converts pyruvate to acetyl-CoA in the overall PDHc reaction, while there has been no such E2o component identified for OGDHc. Instead, the OdhA from Corynebacterium glutamicum and $M t b K g d$ assemble as a single fusion protein with two major domains, the $\mathrm{N}$-terminal acyltransferase-like domain and the E1 domain [83]. It was demonstrated that OdhA from Corynebacterium glutamicum catalyzes both the E1 and E2 reactions and could convert 2-oxoglutarate to succinyl-CoA. However, it is totally dependent on the E2p component of the PDHc as a source of its lipoyl groups. These findings suggested that E1p and OdhA shared the E2p component, in addition to sharing E3, and could form a hybrid complex consisting of E1p, E2p, E3, along with OdhA, in accord with earlier evidence from co-purification experiments [82]. Later, the presence of a hybrid OGDHc/PDHc was also suggested for the Mycobacterium smegmatis $\alpha$ ketoglutarate decarboxylase (MsKGD, E1o) which on reconstitution with dihydrolipoamide acyltransferase (DlaT, the Rv2215 gene product) and E3 revealed OGDHc activity according to formation of succinyl-CoA from OG and CoA [67]. It was proposed that formation of a hybrid OGDHc/PDHc assembly is relevant to stimulation of the MsKGD activity by acetyl-CoA, which is produced by PDHc, and may be important for coordination of the OGDHc/PDHc activities [67,84].

Recently evidence was obtained for a unique property of the human E1a in the L-lysine degradation pathway, where in the absence of a cognate dihydrolipoamide glutaryltransferase component (E2a, no candidate gene has been identified to date), the E1a recruits the E2o and E3 components of the OGDHc for its function in vitro [42,43] and forms a hybrid 2-oxo acid dehydrogenase complex in vivo containing the E1o, E2o and E3 components 
along with E1a [73]. There is also experimental evidence of a direct interaction between the E1a and E2o components: (i) On co-expression of human E1a and E2o in E. coli and in insect Sf9 cells, the E1a $\mathrm{a}_{45-919}$ was co-purified with the E20 68 -453 protein, signaling formation of a high-affinity sub-complex [35]. (ii) Indeed, on titration of the fluorescent N-(1-pyrene) maleimide labeled truncated human E2o (E2 $\mathrm{o}^{1-173}$ di-domain and E2 $\mathrm{o}^{144-386}$ inner core domain) by E1a, dissociation constants in the $1-4 \mu \mathrm{M}$ range were determined, comparable to that for E1o-E2o sub-complex indicating a strong interaction between the E1a and E2o proteins $[35,85]$. (iii) It was further demonstrated that human E1a was co-immunoprecipitated from DBA/2J mouse liver and from control HEK-293 cells with the human E2o, E3 and E1o component as well, supporting the existence of a hybrid 2-oxo acid dehydrogenase complex [73]. The crosstalk between the OGDHc in the TCA cycle and OADHc in L-lysine catabolism is of potential functional relevance since OGDHc can serve as an alternative source of glutaryl-CoA formation in GA1 when E1a function is abolished, which limits the use of E1a-specific inhibitors for substrate-reduction therapy [15,73]. It could furthermore offer a likely explanation for the rather mild or absent phenotype in patients with AMOXAD due to DHTKD1 mutations [9-11]. In addition, the crosstalk may provide a mechanism that could compensate for defects in mitochondrial energy metabolism due to the DHTKD $1^{\mathrm{Y} 486^{*}}$ mutation in CMT2Q [86] and explain an association between DHTKD1 and OGDHL encoded variants and mitochondrial dysfunction related to EoE [6].

\section{Structural Insight into the E1a Active Center}

The idea that a specific lipoyl domain of E2 is recognized by its cognate E1, was generally accepted for a long time. A major recent advance in the field has been the realization that the E1a in the L-lysine degradation pathway could share the same E2o and E3 components with the E1o from the TCA cycle for their function in two distinct metabolic pathways $[42,43,64]$. Next, it was shown that the E1a has about 40 -fold preference in catalytic efficiency with OA versus OG [43], suggesting that the E1a active center has evolved to accommodate the slightly longer OA compared to OG (one additional $\mathrm{CH}_{2}$ group) substrate. These are important determinants for the rational design of E1a inhibitors that could be useful for diseases such as GA1 [36].

Below we discuss the structural insight into the architecture of the human E1a active site during catalysis, as it is a less studied enzyme in the family of 2-oxoacid dehydrogenase complexes. The thiamin diphosphate (ThDP) dependent E1a is the first component of the OADHc [7,9-13], the totality of which carries out the reactions forming glutaryl-CoA according to the overall reaction in Equation (1) and with detailed chemistry in Equations (2)-(5) and Schemes 1 and 2:

$$
\text { 2-oxoadipate }+\mathrm{NAD}^{+}+\mathrm{CoA} \rightarrow \text { glutaryl-CoA }+\mathrm{NADH}+\mathrm{H}^{+}+\mathrm{CO}_{2}
$$

2-oxoadipate + E1a $\rightarrow$ C2-( $\alpha$-hydroxy)- $\gamma$-carboxybutylidene-ThDP-E1a (the enamine intermediate) $+\mathrm{CO}_{2}$

(C2-( $\alpha$-hydroxy)- $\gamma$-carboxybutylidene-ThDP-E1a + lipoyl-E2o $\rightarrow$ S8-glutaryl-dihydrolipoyl-E2o (reductive glutarylation)

$$
\begin{aligned}
& \text { S8-glutaryldihydrolipoyl-E2o }+\mathrm{CoA} \rightarrow \text { glutaryl-CoA + dihydrolipoyl-E2o } \\
& \text { dihydrolipoyl-E2o }+\mathrm{E} 3+\mathrm{NAD}^{+} \rightarrow \text { lipoyl-E2o }+\mathrm{NADH}
\end{aligned}
$$

The recently solved X-ray structures of the human E1a at $1.9 \AA$ (PDB code 6sy1) [35] and at $2.25 \AA$ resolution (PDB code 6U3J) [36] revealed its structural homology to MsKGD $\left(M_{s K G D}{ }_{\Delta 115} ;\right.$ PDB code $2 X T 6 ; 38 \%$ identity) and to $E$. coli $\mathrm{E} 10$ (PDB code 2 jgd; $38 \%$ sequence identity) [66]. The E1a forms a tight homodimer of over $200 \mathrm{kDa}$, common to all ThDPdependent enzymes, burying a more than $5000 \AA^{2}$ area of monomeric accessible surface at the dimer interface (Figure 3A) [36]. Each E1a subunit is structurally composed of four distinct domains displaying the common fold of ThDP-dependent enzymes (Figure 3, top) [35]. The ThDP cofactor is bound between the two subunits and is supported by highly conserved hydrogen bonds and hydrophobic interactions, including Asp333 and Asn366 
from the known ThDP-binding fold (Figure 3B) $[87,88]$. From the reported structural studies several amino acid residues in the E1a active center have been suggested to interact with OA, residues which are not conserved with those involved in OG binding in E1o's: more polar residues including Tyr190 (Phe506 in MsKGD; Phe227 in E. coli E1o), Tyr370 (Phe682 in MsKGD; Phe394 in E. coli E1o), a less bulky Ser263 (Tyr578 in MsKGD; Tyr297 in E. coli E1o), and a conserved Asp707 (Asp1019 in MsKGD; Asp688 in E. coli E1o) [35,36]. To gain further structural insight into the E1a active center, models have been built of its active center with covalent reaction intermediates derived from OA based on the reported X-ray structures of human E1a [35,36] and MsKGD [68], as well as the mechanism of OGDHc with OA presented in Scheme 2. According to the model studies, the pre-decarboxylation covalent adduct at the thiazolium $\mathrm{C} 2$ position of ThDP is stabilized by the salt bridge between its leaving carboxylate group and the imidazole group of His223 (His539 in MsKGD) and by a hydrogen-bonding interaction between the 2-oxo group of OA and the imidazole group of His708 (His1020 in MsKGD) (Figure 3C). The $\varepsilon$-carboxyl group of the OA in the pre-decarboxylation intermediate is sandwiched between Tyr190 and Tyr370 to form polar interactions in accord with structural findings (Figure 3C) [35]. Interestingly, the Tyr190Phe E1a substitution reduced the catalytic efficiency of the human E1a measured in the model reaction with OA by $\sim 5.7$-fold with no activity detected with OG, indicating that Tyr190 is not crucial for OA binding [36]. In comparison, the ThDP-enamine covalent postdecarboxylation intermediate in Figure 3D engaged new hydrogen-bonding interactions where the substrate $\varepsilon$-carboxyl group now interacts with imidazole groups of His 264 and His708 and a side chain hydroxyl group of Ser288, suggesting possible rearrangement of the active site environment (Figure 3D). Earlier, two distinct conformations of the postdecarboxylation intermediate were identified by X-ray crystallography for the MsKGD [68], however, no such information was available for other homologues of E1o so far. Next, on modeling of the E1a active site with the lipoyllysyl-arm of E2o (see Figure 3E for reductive acyl transfer mechanism and Figure 3F), the residues His708 and His 435 are sufficiently proximal to the incoming lipoyllysyl-arm of E2o to act as a general acid catalyst during the reductive glutarylation of E2o, as suggested by the Pan-Jordan model in Scheme 2 [39]. This is also in agreement with findings in P. putida E1b [89], where His $312 \alpha$ and H131 $\beta$ would serve as potential proton donors to the two sulfur atoms of the lipoyllysyl group of E2 during the reductive acyl transfer step. Through amino acid sequence alignment, the P. putida E1b His $312 \alpha$ and H131 $\beta$ residues align well with His435 and His708 in human E1a. An understanding of the possible interactions in the E1o-E2o and E1a-E2o binary sub-complexes at the atomic level remains challenging so far. 

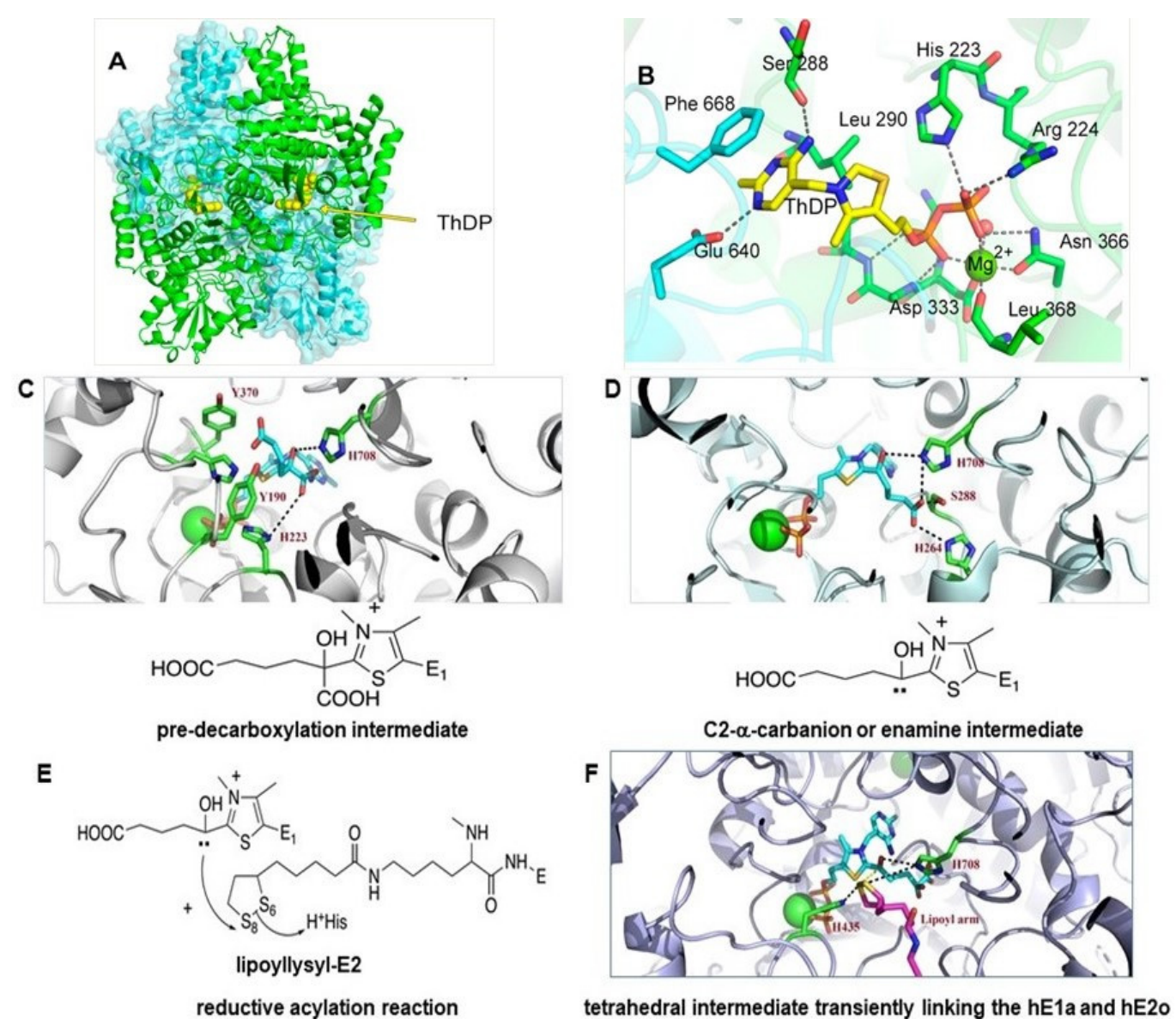

Figure 3. Structure of the human E1a component. (A) Overall fold of the E1a ( $\alpha_{2}$ homodimer) as follows from X-ray structures of the human E1a [35,36]. One monomer is shown in green and the other in cyan and the ThDP cofactor in two active cites is shown in yellow. (B) Active site of E1a with ThDP bound which is stabilized by highly conserved hydrogen bonds and hydrophobic interactions $(\mathbf{A}, \mathbf{B})$ reprinted in part with permission from [36]. (C) Modeling of the E1a active site with predecarboxylation intermediate generated from the nucleophilic attack by the ylide carbanion of ThDP on C2 atom of 2-oxoadipate. (D) Modeling of the E1a active site with the post-decarboxylation intermediate formed after the release of $\mathrm{CO}_{2}$. (E) Schematic representation of acyl transferase reaction between the E1a and E2o components. (F) Modeling of the E1a active site with lipoyllysyl-E2o showing two sulfur atoms of the lipoyllysyl-E2o interacting with $\mathrm{His}^{435}$ and $\mathrm{His}^{708}$ in the active center of E1a. Modeling of the E1a active site in panels $(\mathbf{C}-\mathbf{F})$ were built by using the X-ray structure of E1a (PDB: 6sy1) [35] and MsKGD (PDB: 3zht) [68] and the PyMOL visualization system (v 2.2.1).

\section{Similarities and Differences between the E1o-E2o and E1a-E2o Assembly According to Mass Spectrometric Studies}

To identify the similarities and differences between the E1o-E2o and E1a-E2o subcomplex assembly, protein-protein interactions in the binary E1a-E2o sub-complex were analyzed by HDX-MS. Importantly, the N-terminal region of E1a comprising residues 24-47 experienced significant reduction in deuterium uptake in the E1a-E2o sub-complex (Figure 4A) [85] similar to that observed with the E1o-E2o sub-complex [70]. Also, the region surrounding the ThDP- and $\mathrm{Mg}^{2+}$-binding site, including peptides $485-505,506-518$ and 646-664 in E1a displayed a lower relative deuterium incorporation, suggesting less accessibility to the deuterium on interaction with E2o. However, the peptide comprising residues 407-415 in the same region of E1o displayed modestly increased deuterium uptake $[70,85]$. In Figure $4 \mathrm{~B}$, peptides that become less open for deuterium uptake on interaction with E2o are color-coded in red on the modeled E1 structure. More differences were detected in the interaction of E1a with E2o in the E1a C-terminal region, where peptide 
847-874 was protected from deuterium uptake [85], while peptide comprising residues 865-887 in E1o was exposed to deuterium exchange and displayed a significant increase ( $>1 \mathrm{Da}$ ) in deuterium uptake [70]. These HDX-MS studies led to a major conclusion that, the N-terminal region of both E1o and E1a is a candidate for binding with E2o.

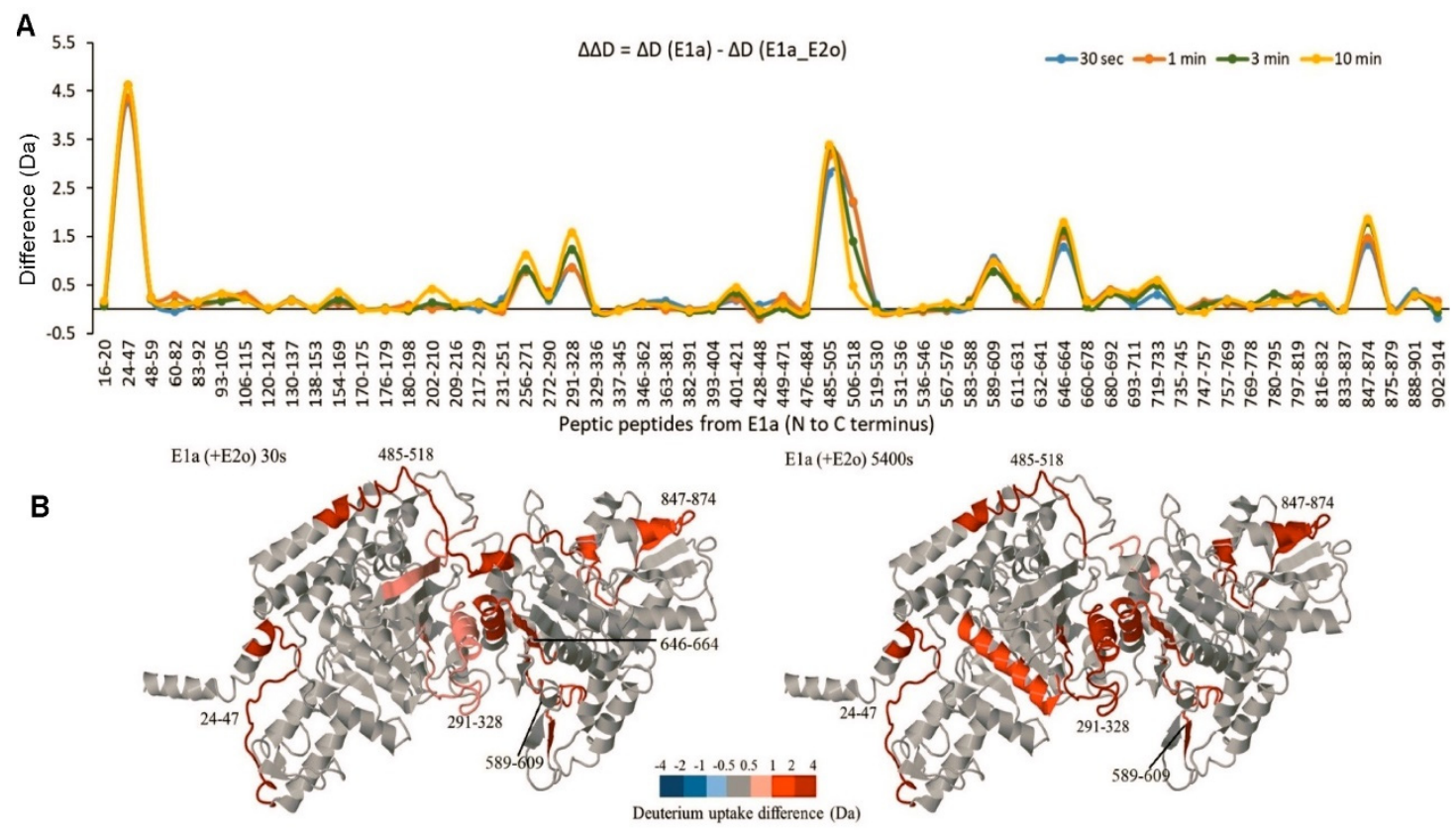

Figure 4. Deuterium uptake of the human E1a peptic peptides on interaction with E2o. (A) Difference plot showing the changes in deuterium incorporation of peptic peptides of $\mathrm{E} 1 \mathrm{a}(\Delta \Delta \mathrm{D}, y$ axis) (deuterons exchanged in peptic peptides of E1a by itself minus deuterons exchanged in the peptic peptides of E1a on interaction with E2o). (B) The E1a peptic peptides protected from HDX on interaction with E2o are color-coded according to the deuterium uptake difference at the first 30s and at 90 min time points [85].

To further elucidate potential loci of interaction in the binary E1a-E2o sub-complex, chemical cross-linking MS (CL-MS) experiments were carried out by using two crosslinkers: $1,1^{\prime}$-carbonyldiimidazole, a zero-length cross-linker (CDI, a spacer length of $2.6 \AA$ and $\mathrm{C} \alpha-\mathrm{C} \alpha$ distance of $\sim 16 \AA$ to be bridged) and disuccinimidyl dibutyric urea (DSBU, $\mathrm{C} \alpha$-C $\alpha$ distance of $\sim 27 \AA$ to be bridged). The majority of cross-linked residues identified in E1a are located in the N-terminal region ( $\mathrm{Lys}^{37}, \mathrm{Lys}^{148}$ and $\mathrm{Lys}{ }^{188}$ ), in the ThDP- $\mathrm{Mg}^{2+}$ binding region (Lys ${ }^{300}$ and Lys ${ }^{450}$ ), and in the C-terminal region $\left(\mathrm{Lys}^{818}\right.$, $\mathrm{Lys}^{826 / 827}$, and Lys $^{852 / 854}$ ) (Figure 5). Lysine residues from those regions were mostly cross-linked to E2o lysine residues from the lipoyl domain $\left(\mathrm{Lys}^{24}, \mathrm{Lys}^{43}, \mathrm{Lys}^{66}, \mathrm{Lys}^{78}\right.$, and $\left.\mathrm{Lys}^{85}\right)$, from the linker region $\left(\mathrm{Lys}^{150}, \mathrm{Lys}^{159}\right)$, and from the inner core domain $\left(\mathrm{Lys}^{240}, \mathrm{Lys}^{286}, \mathrm{Lys}^{342}{ }^{34 y \mathrm{~L}^{371}}\right.$ ) (Figure 5 lower panel). It was notable that a great number of interactions between the C-terminal region of E1a and the lipoyl domain of the E2o have been formed suggesting that the C-terminal region of E1a may play an important role in E1a-E2o interactions, particularly, in the glutaryl transfer from the E1a active center to the E2o. This conclusion was confirmed by studies with the G729R E1a variant identified in AMOXAD disease [85]. Thus, the HDX-MS and CL-MS findings provided several layers of information on E1oE2o and E1a-E2o assembly in Figure 5. First, the studies strongly support the role of the $\mathrm{N}$-terminal region of the human E1o and E1a to interact with E2o, which has now become a general binding mode for E1's with $\alpha_{2}$ quaternary structure [90-93]. Second, in the E2o component, the role of the E2o-core domain in interaction with the E1's was elucidated for the first time. Earlier, this function was assigned mainly to the E1 subunit-binding domain. Third, the role of the C-terminal region of E1a in substrate channeling between the E1a and E2o was identified. 




Figure 5. Inter-component cross-links within the human E1o-E2o and E1a-E2o sub-complex identified by CL-MS by using disuccinimidyl dibutyric urea (DSBU, C $\alpha$-C $\alpha$ distance $~ 27 \AA$ to be bridged) as a cross-linker. The bar plots with cross-link network were generated with xiView visualization tool for xiNET [94].

\section{Structural Modeling of E1a-E2o and E1o-E2o Interactions Using Distance Constraints from}

To gain a deeper understanding of the human E1o-E2o and E1a-E2o interactions in binary sub-complexes, cross-linked guided modeling was performed by using web servers and structural prediction algorithms reported in the literature [94-103]. In the first stage, preliminary models were generated for the human E1o, E1a and E2o by using the I-TASSER algorithm (see Figure 6A for workflow diagram). The E1a structure was built by using the recently reported X-ray structure of the E1a25-919 [36]. The E1o structure was modeled by using the reported X-ray structure of the MsKGD (PDB: 2XT6; 41\% identity) as the search template. The E2o structure was built by using multiple templates with I-TASSER (PDB: 1SCZ; 6H05; 3MAE). In the second stage, the incompatible intra-protein (or intracomponent) cross-links were filtered by using the Xlink Analyzer [98] in visualization system UCSF Chimera (version 1.11) [99] by applying the distance threshold of $35 \AA$ for DSBU and of $25 \AA$ for CDI. The Euclidean cut off was calculated as the sum of the length of the two extended lysine chains $(2 \times 5.5 \AA)$ plus the spacer length $(2.6 \AA$ and $12.5 \AA$ for CDI and DSBU, respectively) with an additional 7.6-12.6 $\AA$ allowed for the conformational dynamics [100]. The compatible intra-component cross-links were utilized as input with an initial model for modeling refinement, which is especially important for proteins with unknown structure, such as human E1o. The utilization of two different cross-linkers can facilitate these refinement steps. The best models of E1o, E1a and E3 were selected according to the confidence score (C-score) and by how well the intra-component crosslinks fit to a model [95]. In the third stage, the resulting protein models were used as input for protein-protein docking by using the HDOCK server [101] and the docking was assisted by the inter-component cross-links identified for the E1a-E2o and E1o-E2o sub-complexes by CL-MS (Figure 5; Figure 6B,C). Also, during the docking stage, the DSBU cross-linked residues were mostly employed as distance restraints since they could provide a broader distance range for protein dynamics compared to the CDI cross-linker (see insert in Figure 6B,C). When the same two Lys residues were found to be cross-linked by both CDI and DSBU, the CDI's distance restraints were employed. The best model was selected from the top 10 solutions by taking into consideration the previous HDX-MS findings and keeping in 
mind the current understanding of the catalytic mechanism of the OGDHc and E1a. First, according to HDX-MS studies, the N-terminal region of E1o (residues 27-40) and of E1a (residues 24-47) displayed significant deuterium uptake decrease upon interaction with E2o, suggesting that the N-terminal region of both proteins may be involved in interaction with E2o. Second, the lipoyl domain of the E2o should be near the E1o/E1a active center at a position such that it can transfer the succinyl/glutaryl group from the E1o/E1a active center to the E2o, thus providing the substrate channeling pathway in accord with the catalytic mechanism. It needs to be noted that the resulting models for the E1o-E2o and E1a-E2o sub-complex assembly in Figure 7 share some similarities.

A

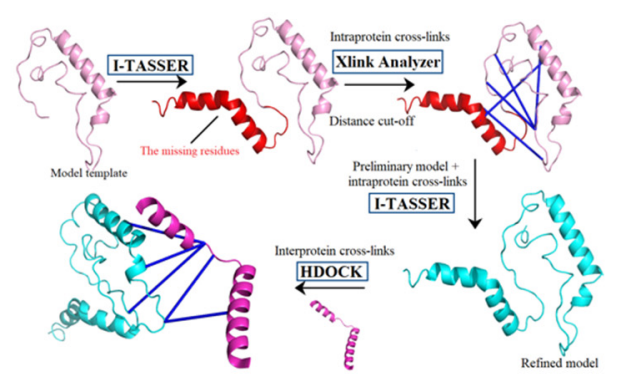

B

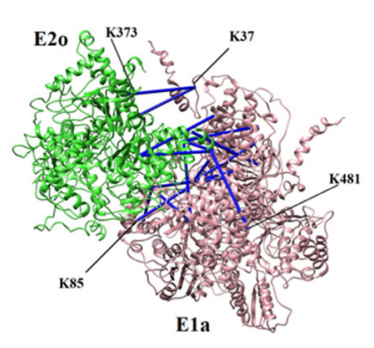

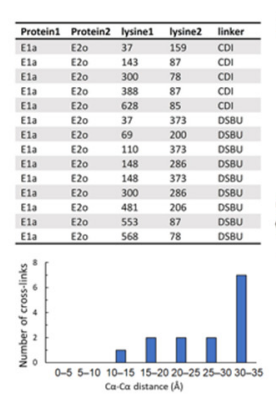

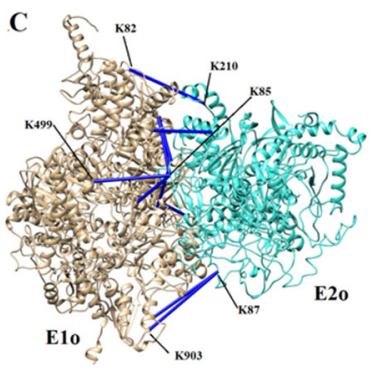

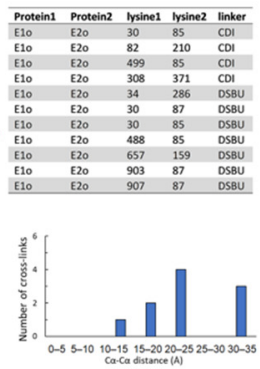

Figure 6. Modeling of the human E1o-E2o and E1a-E2o sub-complex structures. (A) Overview of the modeling workflow using intra-component and inter-component cross-links as residue distance constraints. (B) Mapping of the compatible inter-component cross-links into E1a-E2o sub-complex structure created by protein-protein docking [100]. (C) Mapping of the compatible inter-component cross-links into the E1o-E2o sub-complex structure. The Lys ${ }^{82}$ and Lys ${ }^{87}$ are from different subunits of the E2o trimer [94,104].

Thus, in the E2o protein the inner core region comprising residues 227-453 binds on the 'front side' of the E1o and E1a proteins providing protection for the N-terminal region of the E1o (residues 27-40) and E1a (residues 24-47) from H/D exchange in accord with HDX-MS findings where the N-terminal region of E1o and E1a displayed significant deuterium uptake decrease on interaction with E2o. Also, the lipoyllysyl-arm of the E2o comprising residues 68-128 of the lipoyl domain and residues 129-226 of the linker region preceding the catalytic domain, is swinging around the E1o/E1a ThDP- $\mathrm{Mg}^{2+}$-binding site to provide the catalytic mechanism for succinyl/glutaryl transfer between the E1o/E1a and E2 components. On the other hand, three regions of the E2o core domain with $\alpha$ helix secondary structure comprising residues 191-208 from the linker region and residues 370-386 from the E2o core are located proximal to E1o, while the region comprising residues 273-289 from the E2o core domain points away from the E1o (Figure 7B, Left). In the E1aE2o model, a "clockwise twist" shifts the $\alpha$-helix from the linker region (residues 191-208) away from E1a and brings the $\alpha$-helix from the E2o core domain (residues 273-289) closer to E1a, while the $\alpha$-helix from the E2o core (residues 370-386) approaches the $\mathrm{N}$-terminal region of E1a (Figure 7B, Right). These findings help us to understand how E2o could differentiate between the E1o and E1a and suggest further even more challenging studies to obtain an atomic structural model for the OGDHc and its hybrid complex assembly with E1a. 

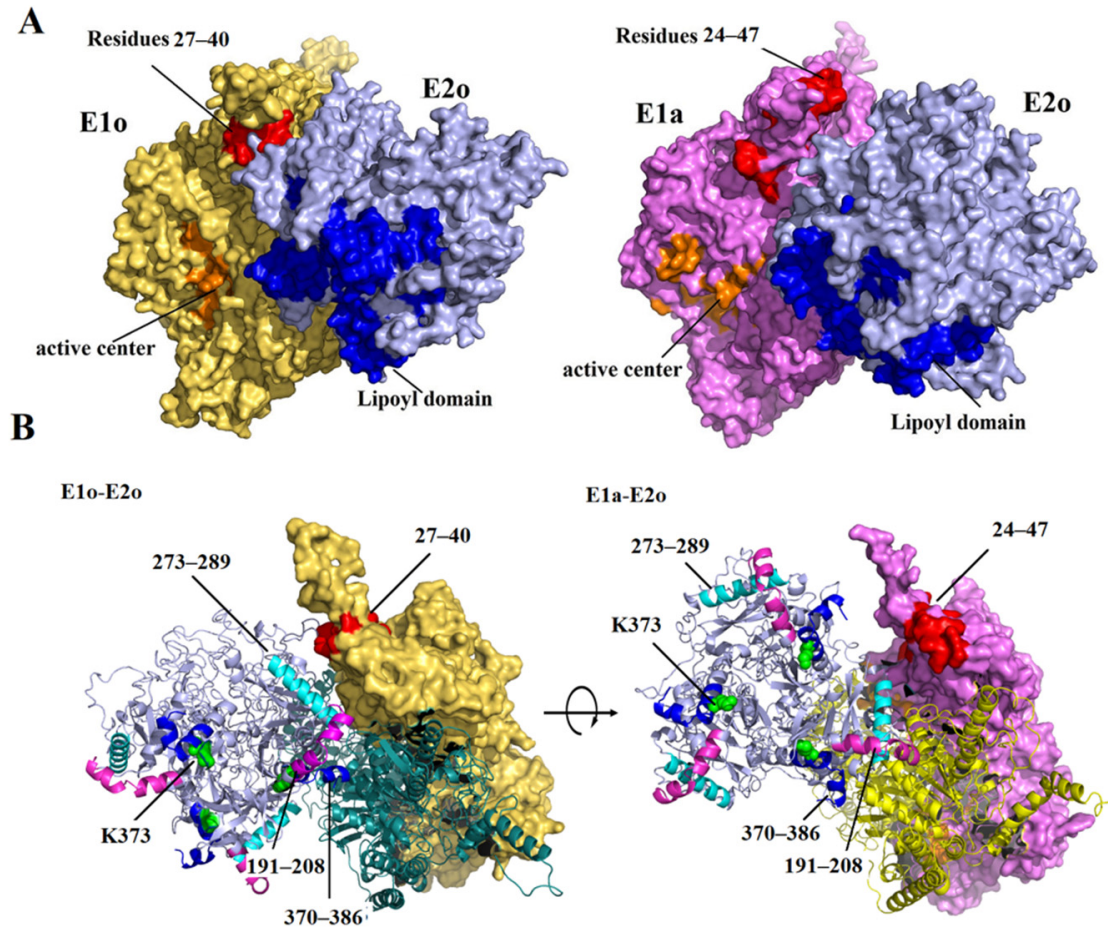

Figure 7. Modeling of the human E1o-E2o and of E1a-E2o sub-complex structures. (A) The E1o $\left(\alpha_{2}\right)$ and the E1a $\left(\alpha_{2}\right)$ homodimers are colored in yellow and in purple, respectively. The E2o core formed by tightly associated trimers is shown in violet. The E2o lipoyl domains are shown in dark blue. The N-terminal residues of E1o (residues 27-40) and E1a (residues 24-47) are shown in red. The E1o and E1a active centers located between the two subunits are shown in orange. (B) View of the E1o-E2o and E1a-E2o sub-complex structures at a different angle. Three regions of the E2o with $\alpha$-helix secondary structure comprising residues 191-208 from the linker region (in blue), and residues 273-289 (in cyan) and 370-386 (in purple) both from the E2o core are indicated showing their different orientation versus E1o (left) and E1a (right) [94,104].

\section{Application of Cryo-EM to Gain Insight into the Architecture of the 2-Oxo Acid Dehydrogenase Complexes}

A thorough identification of protein-protein interactions in the family of the 2-oxo acid dehydrogenase complexes remains a challenge. Early studies of the architecture of the 2-oxo acid dehydrogenase complexes by analysis of electron cryo-microscopy images of the E. coli pyruvate dehydrogenase complex (PDHc) and OGDHc were reported three decades ago [105]. The important observation from these studies was that the E2 components in both complexes exist as 24-mer cubic assemblies that form the structural cores of the complexes. Multiple copies (12-24 subunits) of the E1 and E3 bind to the surface of the E2 core and are separated from the core surface by a gap of $\sim 3-5 \mathrm{~nm}$ [105]. Some 15 years later, the molecular organization of the E. coli PDHc and OGDHc was reinvestigated by using cryo-electron tomography and it was demonstrated that the E1 and E3 components in these complexes are flexibly tethered $\sim 11 \mathrm{~nm}$ away from the E2 core with no significant variations in the E2 core dimensions [106]. Recently, a 3D reconstruction of the human E2o core at $4.7 \AA$ resolution was generated by single-particle cryo-EM [35]. The EM reconstruction showed 24 C-terminal core domains grouped as eight trimers and positioned at each of the eight vertices of the cubic cage with octahedral symmetry [35]. There was no electron density found for the other parts of the E2o, including the N-terminal lipoyl domain and much of the linker region, suggesting that those regions are highly flexible and dynamic [35]. The human E2o core structure generated was similar those reported earlier for the E. coli E2o core [47], the E. coli E2p core [52] and for the A. vinelandii E2p core [49], all obtained by $\mathrm{X}$-ray crystallography. In the 3D reconstruction of the co-expressed human E1a-E2o sub-complexes by single-particle cryo-EM, there was no density evident for the E1a protein, 
indicating that their interaction could be short lived [35]. A molecular weight of the human $\mathrm{E}^{1} \mathrm{a}_{45-919}-\mathrm{E}_{2} \mathrm{O}_{68-453}$ sub-complex of $2.45 \mathrm{MDa}$ was derived from small-angle $\mathrm{X}$-ray scattering (SAXS) experiments, suggesting a stoichiometry of one E1a dimer per one E2o trimer for their binding [35], similar to that previously deduced for the human E1o-E2o sub-complex [70].

In contrast to 2-oxoglutarate dehydrogenase complexes, cryo-EM studies of the pyruvate dehydrogenase complexes (PDHc's) from Gram-positive bacteria such as Bacillus stearothermophilus [107], yeast [108-110], bovine PDHc [111,112], and human PDHc [51,79,80] revealed a 60-mer E2p core with the morphology of a pentagonal dodecahedron and icosahedral symmetry. The first reconstruction of a three-dimensional structure of E2p was reported for the icosahedral truncated E2p ( $t \mathrm{E} 2 \mathrm{p})$ core from Saccharomyces cerevisiae (S. cerevisiae) which lacks the lipoyl domain and the E1-binding domain $[108,109]$. The cryo-EM structure of the $S$. cerevisiae $t \mathrm{E} 2 \mathrm{p}$ core generated at $25 \AA$ resolution revealed that the E2p catalytic domains are arranged in cone-shaped trimers at each of the 20 vertices of the dodecahedron structure which are interconnected by 30 bridges [108,109]. The cryo-EM studies also revealed flexibility (size variability) in the arrangement of the $S$. cerevisiae E2p core that is due to conformational changes in the trimers and in the bridges that connect adjacent trimers suggesting a flexible "breathing" core, which may be important for multienzyme complex function, such as channeling of intermediates between the active centers of the individual components [112]. Next, a 3-D reconstruction of the S. cerevisiae E2p core in sub-complex with E1p's generated by cryo-EM at $\sim 30 \AA$ resolution suggested that the length of the E2p inner linkers that anchors the E1p tetramers from the outer shell to the E2p core also may vary from $\sim 50-75 \AA$ depending on the occupancy of the outer shell by E1p's, contributing to the overall dynamics of the S. cereviciae PDHc [110]. The $\sim 70 \AA$ resolution structure generated for the E1p outer shell was significantly lower compared to the E2p core and was less reliable for interpretation of the E1p's arrangement around the E2p core [110]. The high resolution cryo-EM and single-particle reconstruction enabled definition of 3D structures of the human full-length E2p at $15 \AA$ resolution and of the $t \mathrm{E} 2 \mathrm{p}$ core (devoid of lipoyl domains and E1p-binding domains) at $8.8 \AA$ and $3.1 \AA$ resolution that also enabled visualization of the secondary structure elements within the $t \mathrm{E} 2 \mathrm{p}$ core, including the $\alpha$ helices and $\beta$ sheets [51,79]. The reconstructed structures were similar and consisted of 60 inner core domains arranged as 20 trimers into pentagonal dodecahedron. However, the E2p linker region that connects E1p's to the E2p core domain was invisible due to its high flexibility [79]. The human E2p core similar to that for S. cerevisiae E2p core, exhibited variation in particle size suggesting its flexibility [51].

In eukaryotes, the complexity of the E2p core is increased, being formed by the E2p and E3-binding protein (E3BP) components, which assemble into a 60-mer central core with icosahedral symmetry [113]. Two alternative models have been suggested for assembly of E2/E3BP: 48 copies of E2 and 12 copies of E3BP or 40 copies of E2 and 20 copies of E3BP, where the E3BP substitutes for E2 within trimers [79]. The homology model of the E3BP inner core supports the structure of the preformed heterotrimer with one copy of E3BP and two copies of E2 [79]. Recently, the structure of the recombinant human E2-E3BP core was determined by cryo-EM at $6.3 \AA$ resolution [80]. The final 3D map showed the E2-E3BP inner core where the E2 and E3BP subunits remained undistinguished. The $\mathrm{N}$-terminal lipoyl domains, E1 binding domain and linker regions were invisible due to their high conformational flexibility. The negative stain electron microscopy, native and cross-linking MS gave evidence of the possible structural asymmetry in distribution of the E1p and E3p components at the periphery of the E2p core that suggested a "division-oflabor"mechanism that could be modulated by the presence of substrate [80]. Currently, it is evident that even high-resolution structures of human E2p core and E2p-E3BP core assembly obtained by cryo-EM cannot provide an ultimate answer to protein-protein interactions in 2-oxo acid dehydrogenase complexes. To address these issues, integrative structural approaches will be required. 


\section{Conclusions and Perspectives}

The 2-oxoacid dehydrogenase complexes are macromolecular assemblies functioning in different metabolic pathways. These assemblies provide high catalytic efficiency and high-level of regulation to the metabolic pathways in the cell and the regulation of gene expression in the nucleus. Recent experimental findings in vitro and in vivo have identified the formation of hybrid complexes between the OGDHc and E1a, suggesting functional crosstalk between the two distinct metabolic pathways and an additional layer of regulation that needs to be thoroughly established. The direct association of the OGDHc and/or OADHc with known histone acetyltransferase KAT2 and histone H3/H4 has recently been discovered, suggesting a functional crosstalk between the KAT2 and OGDHc/OADHc in nuclei for the purpose of posttranslational modification of histones by succinylation/glutarylation and regulation of gene expression, tumor cell proliferation, and tumor formation $[31,32]$. However, direct involvement of the OGDH and OADHc in succinylation/glutarylation of mitochondrial and nuclear proteins needs to be explored. While over the past several years the importance of the OGDHc and OADHc in human health and disease, and their role as potential therapeutic targets have been increasingly emphasized, recent studies defining their structural organization and assembly into macromolecular machines are limited by cryo-EM reconstruction of the E2o inner core. In the absence of atomic resolution structure of any of the 2-oxo acid dehydrogenase complexes, recent advances in chemical cross-linking coupled with mass spectrometry, and in computational data processing have emerged as powerful tools to elucidate the potential interactions in binary E1o-E2o and E1a-E2o assemblies, and to identify their binding surfaces as well as their relative orientation via molecular modeling. Recent advances in negative stain EM and in single particle cryo-EM are promising to determine the pseudo-atomic structural models for assembly in 2-oxoacid dehydrogenase complexes. The combination of biochemical, biophysical and structural approaches by using cross-linking MS and cryo-EM structural biology is particularly promising to provide vital information for the assembly of 2-oxoacid dehydrogenase complexes, their function and regulation. At the same time, such studies will create a scaffold onto which novel inhibitors could be designed to mitigate the consequences of diseases associated with dysfunction of the TCA cycle or lysine degradation.

Author Contributions: Conceptualization, N.S.N., S.M.H., F.J.; writing-original draft preparation, N.S.N., X.Z., J.L.; J.Z., L.Y.; writing-review and editing, N.S.N., S.M.H., F.J.; funding, F.J., S.M.H. All authors have read and agreed to the published version of the manuscript.

Funding: This work was supported by National Institutes of Health Grant R15GM116077-01 (to F.J.), National Science Foundation Grant CHE-1402675 (to F.J.), the Eunice Kennedy Shriver National Institute of Child Health \& Human Development of the National Institutes of Health (NIH) under Award Number R03HD092878 (to S.M.H.) and R21HD088775 (to S.M.H.), and Rutgers-Newark Chancellor's SEED Grants (to F.J.).

Institutional Review Board Statement: Not applicable.

Informed Consent Statement: Not applicable.

Data Availability Statement: The mass spectrometry proteomics data have been deposited to the ProteomeXchange Consortium (www.proteomexchange.org) via the PRIDE partner repository with the dataset identifier PXD017792 (accessed on 21 April 2020) and PXD023525 (not announced yet).

Acknowledgments: Authors are very grateful to the many scientists who have contributed to our understanding of the mechanism and structure of the 2-oxo acid dehydrogenase complexes and for challenges undertaken to understand the protein-protein interactions in the 2-oxo acid dehydrogenase complexes.

Conflicts of Interest: The authors declare no conflict of interest. The funders had no role in the design of the study; in the collection, analyses, or interpretation of data; in the writing of the manuscript, or in the decision to publish the results. 


\begin{abstract}
Abbreviations
OGDHc, 2-oxoglutarate dehydrogenase complex; PDHc, pyruvate dehydrogenase complex; OADHc, 2-oxoadipate dehydrogenase complex; E1o, 2-oxoglutarate dehydrogenase, the first component of the OGDHc; E1a, 2-oxoadipate dehydrogenase; E2o, dihydrolipoamide succinyltransferase, the second component of the OGDHc; E3, dihydrolipoamide dehydrogenase, the third component of the OGDHc; LDo, lipoyl domain; CDo, E2o catalytic domain; PSBD, peripheral subunit binding domain; E3BP, E3-binding protein; DHTKD1, gene encoding the E1a; h, human; E. coli, Escherichia coli; S. cerevisiae, Saccharomyces cerevisiae; Mtb, Mycobacterium tuberculosis; B. stearothermophilus, Bacillus stearothermophilus; Ms, Mycobacterium smegmatis; SIRT5, sirtuin 5; PTMs, post-translational modifications; TCA cycle, tricarboxylic acid cycle; ThDP, thiamin diphosphate; AMOXAD, alpha-aminoadipic and alpha-ketoadipic aciduria; CMT2Q, Charcot-Marie-Tooth disease type 2Q; GA1, glutaric aciduria type 1; EoE, eosinophilic esophagitis; HDX-MS, hydrogen-deuterium exchange mass spectrometry; CL-MS, chemical cross-linking mass spectrometry; FT-MS, Fourier transform mass spectrometry; cryo-EM, cryogenic electron microscopy; CDI, 1,1'-carbonyldiimidazole cross-linker; DSBU or Bu$\mathrm{UrBu}$, disuccinimidyl dibutyric urea; PDB, Protein Data Bank.
\end{abstract}

\title{
References
}

1. Gibson, G.E.; Park, L.C.; Sheu, K.-F.R.; Blass, J.P.; Calingasan, N.Y. The $\alpha$-ketoglutarate dehydrogenase complex in neurodegeneration. Neurochem. Int. 2000, 36, 97-112. [CrossRef]

2. Gibson, G.; Kingsbury, A.; Xu, H.; Lindsay, J.; Daniel, S.; Foster, O.; Lees, A.; Blass, J. Deficits in a tricarboxylic acid cycle enzyme in brains from patients with Parkinson's disease. Neurochem. Int. 2003, 43, 129-135. [CrossRef]

3. Gibson, G.E.; Xu, H.; Chen, H.-L.; Chen, W.; Denton, T.T.; Zhang, S. Alpha-ketoglutarate dehydrogenase complex-dependent succinylation of proteins in neurons and neuronal cell lines. J. Neurochem. 2015, 134, 86-96. [CrossRef] [PubMed]

4. Chen, H.; Denton, T.T.; Xu, H.; Calingasan, N.; Beal, M.F.; Gibson, G.E. Reductions in t mitochondrial enzyme $\alpha$-ketoglutarate dehydrogenase complex in neurodegenerative disease-beneficial or detrimental? J. Neurochem. 2016, 139, 823-838. [CrossRef] [PubMed]

5. Yap, Z.Y.; Strucinska, K.; Matsuzaki, S.; Lee, S.; Si, Y.; Humphries, K.; Tarnopolsky, M.A.; Yoon, W.H. A biallelic pathogenic variant in the OGDH gene results in a neurological disorder with features of a mitochondrial disease. J. Inherit. Metab. Dis. 2021, 44, 388-400. [CrossRef] [PubMed]

6. Sherrill, J.D.; Kc, K.; Wang, X.; Wen, T.; Chamberlin, A.; Stucke, E.M.; Collins, M.H.; Abonia, J.P.; Peng, Y.; Wu, Q.; et al. Whole-exome sequencing uncovers oxidoreductases DHTKD1 and OGDHL as linkers between mitochondrial dysfunction and eosinophilic esophagitis. JCI Insight 2018, 3, 99922. [CrossRef] [PubMed]

7. Bunik, V.I.; Degtyarev, D. Structure-function relationships in the 2-oxo acid dehydrogenase family: Substrate-specific signatures and functional predictions for the 2-oxoglutarate dehydrogenase-like proteins. Proteins Struct. Funct. Bioinform. 2008, 71, 874-890. [CrossRef]

8. Yoon, W.H.; Sandoval, H.; Nagarkar-Jaiswal, S.; Jaiswal, M.; Yamamoto, S.; Haelterman, N.A.; Putluri, N.; Putluri, V.; Sreekumar, A.; Tos, T.; et al. Loss of Nardilysin, a Mitochondrial Co-chaperone for $\alpha$-Ketoglutarate Dehydrogenase, Promotes mTORC1 Activation and Neurodegeneration. Neuron 2017, 93, 115-131. [CrossRef]

9. Danhauser, K.; Sauer, S.W.; Haack, T.B.; Wieland, T.; Staufner, C.; Graf, E.; Zschocke, J.; Strom, T.M.; Traub, T.; Okun, J.G.; et al. DHTKD1 Mutations Cause 2-Aminoadipic and 2-Oxoadipic Aciduria. Am. J. Hum. Genet. 2012, 91, 1082-1087. [CrossRef]

10. Stiles, A.R.; Venturoni, L.; Mucci, G.; Elbalalesy, N.; Woontner, M.; Goodman, S.I.; Abdenur, J.E. New Cases of DHTKD1 Mutations in Patients with 2-Ketoadipic Aciduria. JIMD Rep. 2015, 25, 15-19. [CrossRef]

11. Hagen, J.; Brinke, H.T.; Wanders, R.J.A.; Knegt, A.C.; Oussoren, E.; Hoogeboom, A.J.M.; Ruijter, G.J.G.; Becker, D.; Schwab, K.O.; Franke, I.; et al. Genetic basis of alpha-aminoadipic and alpha-ketoadipic aciduria. J. Inherit. Metab. Dis. 2015, 38, 873-879. [CrossRef]

12. Xu, W.Y.; Gu, M.M.; Sun, L.H.; Guo, W.T.; Zhu, H.B.; Ma, J.F.; Yuan, W.T.; Kuang, Y.; Ji, B.J.; Wu, X.L.; et al. A nonsence mutation in DHTKD1 causes Charcot-Marie-Tooth type 2 in a large chinese pedigree. Am. J. Hum. Genet. 2012, 91, 1088-1094. [CrossRef]

13. Xu, W.; Zhu, H.; Gu, M.; Luo, Q.; Ding, J.; Yao, Y.; Chen, F.; Wang, Z. DHTKD1 is essential for mitochondrial biogenesis and function maintenance. FEBS Lett. 2013, 587, 3587-3592. [CrossRef] [PubMed]

14. Xu, W.-Y.; Zhu, H.; Shen, Y.; Wan, Y.-H.; Tu, X.-D.; Wu, W.-T.; Tang, L.; Zhang, H.-X.; Lu, S.-Y.; Jin, X.-L.; et al. DHTKD1 deficiency causes Charcot-Marie-Tooth disease in mice. Mol. Cell. Biol. 2018, 38. [CrossRef] [PubMed]

15. Biagosch, C.; Ediga, R.D.; Hensler, S.-V.; Faerberboeck, M.; Kuehn, R.; Wurst, W.; Meitinger, T.; Kölker, S.; Sauer, S.; Prokisch, H. Elevated glutaric acid levels in Dhtkd1-/Gcdh-double knockout mice challenge our current understanding of lysine metabolism. Biochim. Biophys. Acta Mol. Basis Dis. 2017, 1863, 2220-2228. [CrossRef]

16. Vatrinet, R.; Leone, G.; De Luise, M.; Girolimetti, G.; Vidone, M.; Gasparre, G.; Porcelli, A.M. The $\alpha$-ketoglutarate dehydrogenase complex in cancer metabolic plasticity. Cancer Metab. 2017, 5, 1-14. [CrossRef] 
17. Burr, S.P.; Costa, A.S.; Grice, G.L.; Timms, R.T.; Lobb, I.T.; Freisinger, P.; Dodd, R.B.; Dougan, G.; Lehner, P.J.; Frezza, C.; et al. Mitochondrial Protein Lipoylation and the 2-Oxoglutarate Dehydrogenase Complex Controls HIF1 $\alpha$ Stability in Aerobic Conditions. Cell Metab. 2016, 24, 740-752. [CrossRef]

18. Lu, X.; Yang, P.; Zhao, X.; Jiang, M.; Hu, S.; Ouyang, Y.; Zeng, L.; Wu, J. OGDH mediates the inhibition of SIRT5 on cell proliferation and migration of gastric cancer. Exp. Cell Res. 2019, 382, 111483. [CrossRef]

19. Lu, X.; Wu, N.; Yang, W.; Sun, J.; Yan, K.; Wu, J. OGDH promotes the progression of gastric cancer by regulating mito-chondrial bioenergetics and Wnt/ $\alpha$-catenin signal pathway. Onco. Targets 2019, 12, 7489-7500. [CrossRef]

20. Allen, E.L.; Ulanet, D.B.; Pirman, D.; Mahoney, C.E.; Coco, J.; Si, Y.; Chen, Y.; Huang, L.; Ren, J.; Choe, S.; et al. Differential Aspartate Usage Identifies a Subset of Cancer Cells Particularly Dependent on OGDH. Cell Rep. 2016, 17, 876-890. [CrossRef] [PubMed]

21. Anderson, N.M.; Li, D.; Peng, H.L.; Laroche, F.J.F.; Mansour, M.R.; Gjini, E.; Aioub, M.; Helman, D.J.; Roderick, J.E.; Cheng, T.; et al. The TCA cycle transferase DLST is important for MYC-mediated leukemogenesis. Leukemia 2016, 30, 1365-1374. [CrossRef] [PubMed]

22. Ilic, N.; Birsoy, K.; Aguirre, A.J.; Kory, N.; Pacold, M.E.; Singh, S.; Moody, S.E.; DeAngelo, J.D.; Spardy, N.A.; Freinkman, E.; et al PIK3CA mutant tumors depend on oxoglutarate dehydrogenase. Proc. Natl. Acad. Sci. USA 2017, 114, E3434-E3443. [CrossRef] [PubMed]

23. Anderson, N.M.; Mucka, P.; Kern, J.G.; Feng, H. The emerging role and targetability of the TCA cycle in cancer metabolism Protein Cell 2018, 9, 216-237. [CrossRef] [PubMed]

24. Zhang, Z.; Tan, M.; Xie, Z.; Dai, L.; Chen, Y.; Zhao, Y. Identification of lysine succinylation as a new post-translational modification. Nat. Chem. Biol. 2010, 7, 58-63. [CrossRef]

25. Tan, M.; Peng, C.; Anderson, K.A.; Chhoy, P.; Xie, Z.; Dai, L.; Park, J.; Chen, Y.; Huang, H.; Zhang, Y.; et al. Lysine glutarylation is a protein post- translational modification regulated by SIRT5. Cell Metab. 2014, 19, 605-617. [CrossRef]

26. Rardin, M.J.; He, W.; Nishida, Y.; Newman, J.C.; Carrico, C.; Danielson, S.R.; Guo, A.; Gut, P.; Sahu, A.K.; Li, B.; et al. SIRT5 Regulates the Mitochondrial Lysine Succinylome and Metabolic Networks. Cell Metab. 2013, 18, 920-933. [CrossRef]

27. Yang, Y.; Gibson, G.E. Succinylation Links Metabolism to Protein Functions. Neurochem. Res. 2019, 44, 2346-2359. [CrossRef]

28. Xie, Z.; Dai, J.; Dai, L.; Tan, M.; Cheng, Z.; Wu, Y.; Boeke, J.D.; Zhao, Y. Lysine Succinylation and Lysine Malonylation in Histones. Mol. Cell. Proteom. 2012, 11, 100-107. [CrossRef]

29. Schmiesing, J.; Schlüter, H.; Ullrich, K.; Brauke, T.; Mühlhausen, C. Interaction of glutaric aciduria type 1-related glutar-yl-CoA dehydrogenase with mitochondrial matrix proteins. PLoS ONE 2014, 9, e87715. [CrossRef] [PubMed]

30. Schmiesing, J.; Storch, S.; Dörfler, A.C.; Schweizer, M.; Makrypidi-Fraune, G.; Thelen, M.; Sylvester, M.; Gieselmann, V.; Koch-Nolte, F.; Tidow, H.; et al. Disease-linked glutarylation in pairs function and interactions of mitochondrial proteins and contributes to mitochondrial heterogeneity. Cell Rep. 2018, 24, 2946-2956. [CrossRef]

31. Bao, X.; Liu, Z.; Zhang, W.; Gladysz, K.; Fung, Y.M.E.; Tian, G.; Xiong, Y.; Wong, J.W.H.; Yuen, K.W.Y.; Li, X.D. Glutarylation of histone H4 Lysine 91 regulates chromatin dynamics. Mol. Cell 2019, 76, 660-675. [CrossRef]

32. Wang, Y.; Guo, Y.R.; Liu, K.; Yin, Z.; Liu, R.; Xia, Y.; Tan, L.; Yang, P.; Lee, J.H.; Li, X.; et al. KAT2A coupled with the $\alpha$-KGDH complex acts as a histone $\mathrm{H}_{3}$ succinyltransferase. Nature 2017, 552, 273-277. [CrossRef] [PubMed]

33. Nemeria, N.S.; Gerfen, G.; Reddy Nareddy, P.; Yang, L.; Zhang, X.; Szostak, M.; Jordan, F. The mitochondrial 2-oxoadipate and 2-oxoglutarate dehydrogenase complexes share their E2 and E3 components for their function and both generate reactive oxygen species. Free Radic. Biol. Med. 2018, 115, 136-145. [CrossRef] [PubMed]

34. Liu, Y.; You, Y.; Lu, Z.; Yang, J.; Li, P.; Liu, L.; Xu, H.; Niu, Y.; Cao, X. N6-methyl- adenosine RNA modification-mediated cellular metabolism rewiring inhibits viral replication. Science 2019, 365, 1171-1176. [CrossRef] [PubMed]

35. Bezerra, G.A.; Foster, W.R.; Bailey, H.J.; Hicks, K.G.; Sauer, S.W.; Dimitrov, B.; McCorvie, T.J.; Okun, J.G.; Rutter, J.; Kölker, S.; et al. Crystal structure and interaction studies of human DHTKD1 provide insight into a mitochondrial megacomplex in lysine catabolism. Iucr. J. 2020, 7, 693-706. [CrossRef] [PubMed]

36. Leandro, J.; Khamrui, S.; Wang, H.; Suebsuwong, C.; Nemeria, N.; Huynh, K.; Moustakim, M.; Secor, C.; Wang, M.; Dodatko, T.; et al. Inhibition and crystal structure of the human DHTKD1-Thiamin diphosphate complex. ACS Chem. Biol. 2020, 15, $2041-2047$. [CrossRef]

37. Jordan, F. Current mechanistic understanding of thiamin diphosphate-dependent enzymatic reactions. Nat. Prod. Rep. 2003, 20, 184-201. [CrossRef]

38. Frey, P.A.; Fluornoy, D.S.; Gruys, K.; Yang, Y.S. Intermediates in reductive trans acetylation catalyzed by pyruvate dehydrogenase complex. Ann. N. Y. Acad. Sci. 1989, 573, 21-35. [CrossRef]

39. Pan, K.; Jordan, F. D,L-S-methyllipoic acid methyl ester, a kinetically viable model for S- protonated lipoic acid as the oxidizing agent in reductive acyl transfers catalyzed by the 2-oxoacid dehydrogenase multienzyme complexes. Biochemistry 1998, 37, 1357-1364. [CrossRef]

40. Balakrishnan, A.; Nemeria, N.; Chakraborty, S.; Kakalis, L.; Jordan, F. Determination of pre- steady-state rate constants on the Eschericia coli pyruvate dehydrogenase complex reveals that loop movement controls the rate-limiting step. J. Am. Chem. Soc. 2012, 134, 18644-18655. [CrossRef]

41. Chakraborty, J.; Nemeria, N.S.; Farinas, E.; Jordan, F. Catalysis of transthiolacylation in the active centers of dihy-drolipoamide acyltransferase components of 2-oxo acid dehydrogenase complexes. FEBS Open Bio. 2018, 8, 880-896. [CrossRef] [PubMed] 
42. Nemeria, N.S.; Gerfen, G.; Yang, L.; Zhang, X.; Jordan, F. Evidence for functional and regulatory cross-talk between the tricarboxylic acid cycle 2-oxoglutarate dehydrogenase complex and 2-oxoadipate dehydrogenase on the 1-lysine, 1-hydroxylysine and l-tryptophan degradation pathways from studies in vitro. Biochim. Biophys. Acta Bioenerg. 2018, 1859, 932-939. [CrossRef] [PubMed]

43. Shim, D.J.; Nemeria, N.S.; Balakrishnan, A.; Patel, H.; Song, A.; Wang, J.; Jordan, F.; Farinas, E. Assignment of function to Histidines 260 and 298 by engineering the E1 component of the Escherichia coli 2-oxoglutarate dehydrogenase complex; substitutions that lead to acceptance of substrates lacking the 5-carboxyl group. Biochemistry 2011, 50, 7705-7709. [CrossRef] [PubMed]

44. Jones, D.; Stott, K.M.; Reche, P.A.; Perham, R.N. Recognition of the lipoyl domain is the ultimate determinant of substrate channelling in the pyruvate dehydrogenase multienzyme complex. J. Mol. Biol. 2001, 305, 49-60. [CrossRef] [PubMed]

45. Jones, D.D.; Perham, R.N. The role of loop and beta-turn residues as structural and functional determinants for the lipoyl domain from the Eschericia coli 2-oxoglutarate dehydrogenase complex. Biochem. J. 2008, 409, 357-366. [CrossRef] [PubMed]

46. Fries, M.; Stott, K.M.; Reynolds, S.; Perham, R.N. Distinct Modes of Recognition of the Lipoyl Domain as Substrate by the E1 and E3 Components of the Pyruvate Dehydrogenase Multienzyme Complex. J. Mol. Biol. 2007, 366, 132-139. [CrossRef]

47. Knapp, J.E.; Mitchell, D.T.; Yazdi, M.A.; Ernst, S.R.; Reed, L.J.; Hackert, M.L. Crystal structure of the truncated cubic core component of the Escherichia coli 2-oxoglutarate dehydrogenase multienzyme complex. J. Mol. Biol. 1998, 280, 655-668. [CrossRef]

48. Knapp, J.E.; Carroll, D.; Lawson, J.E.; Ernst, S.R.; Reed, L.J.; Hackert, M.L. Expression, purification, and structural analysis of the trimeric form of the catalytic domain of the Escherichia coli dihydrolipoamide succinyl transferase. Protein Sci. 2008, 9, 37-48. [CrossRef]

49. Mattevi, A.; Obmolova, G.; Kalk, K.H.; Westphal, A.H.; de Kok, A.; Hol, W.G. Refined crystal structure of the catalytic domain of dihydrolipoyl transacetylase (E2p) from Azotobacter vinelandiiat 2.6̊ resolution. J. Mol. Biol. 1993, 230, 1183-1199. [CrossRef]

50. Kato, M.; Wynn, R.M.; Chuang, J.L.; Brautigam, C.A.; Custorio, M.; Chuang, D.T. A synchronized substrate-gating mechanism revealed by cubic-core structure of the bovine branched-chain $\alpha$-ketoacid dehydrogenase complex. EMBO J. 2006, 25, 5983-5994. [CrossRef]

51. Yu, X.; Hiromasa, Y.; Tsen, H.; Stoops, J.K.; Roche, T.E.; Zhou, Z.H. Structures of the Human Pyruvate Dehydrogenase Complex Cores: A Highly Conserved Catalytic Center with Flexible N-Terminal Domains. Structure 2008, 16, 104-114. [CrossRef] [PubMed]

52. Wang, J.; Nemeria, N.S.; Chandrasekhar, K.; Kumaran, S.; Arjunan, P.; Reynolds, S.; Calero, G.; Brukh, R.; Kakalis, L.; Furey, W.; et al. Structure and Function of the Catalytic Domain of the Dihydrolipoyl Acetyltransferase Component in Escherichia coli Pyruvate Dehydrogenase Complex. J. Biol. Chem. 2014, 289, 15215-15230. [CrossRef] [PubMed]

53. Lewendon, A.; Murray, L.A.; Shaw, W.V. Replacement of catalytic histidine-195 of chloramphenicl acetyltransferase: Evidence for general base role for glutamate. Biochemistry 1994, 33, 1944-1950. [CrossRef]

54. Guest, J.R. Functional implications of structural homologies between chloramphenicol acetyltransferase and dihy-drolipoamide acetyltransferase. FEMS Microbiol. Let. 1987, 44, 417-422. [CrossRef]

55. Shaw, W.V.; Leslie, A.G.W. Chloramphenicol Acetyltransferase. Annu. Rev. Biophys. Biophys. Chem. 1991, 20, 363-386. [CrossRef] [PubMed]

56. Starkov, A.A.; Fiskum, G.; Chinopoulos, C.; Lorenzo, B.J.; Browne, S.E.; Patel, M.S.; Beal, M.F. Mitochondrial $\alpha$-ketoglutarate dehydrogenase complex generates reactive oxygen species. J. Neurosci. 2004, 24, 7779-7788. [CrossRef]

57. Tretter, L.; Adam-Vizi, V. Generation of reactive oxygen species in the reaction catalyzed by $\alpha$-ketoglutarate dehy-drogenase. J. Neurosci. 2004, 24, 7771-7778. [CrossRef] [PubMed]

58. Tretter, L.; Adam-Vizi, V. $\alpha$-Ketoglutarate dehydrogenase: A target and generator of oxidative stress. Philos. Trans. R. Soc. Lond. B Biol. Sci. 2005, 360, 2335-2345. [CrossRef]

59. Quinlan, C.L.; Goncalves, R.L.S.; Hey-Mogensen, M.; Yadava, N.; Bunik, V.I.; Brand, M.D. The 2-Oxoacid Dehydrogenase Complexes in Mitochondria Can Produce Superoxide/Hydrogen Peroxide at Much Higher Rates Than Complex I. J. Biol. Chem. 2014, 289, 8312-8325. [CrossRef]

60. Nemeria, N.S.; Ambrus, A.; Patel, H.; Gerfen, G.; Adam-Vizi, V.; Tretter, L.; Zhou, J.; Wang, J.; Jordan, F. Human 2-Oxoglutarate Dehydrogenase Complex E1 Component Forms a Thiamin-derived Radical by Aerobic Oxidation of the Enamine Intermediate. J. Biol. Chem. 2014, 289, 29859-29873. [CrossRef]

61. Ambrus, A.; Nemeria, N.S.; Torocsik, B.; Tretter, L.; Nilsson, M.; Jordan, F.; Adam-Vizi, V. Formation of reactive oxygen species by human and bacterial pyruvate and 2-oxoglutarate dehydrogenase multienzyme complexes reconstituted from re-combinant components. Free Radic. Biol. Med. 2015, 89, 642-650. [CrossRef] [PubMed]

62. Goncalves, R.L.; Bunik, V.I.; Brand, M.D. Production of superoxide/hydrogen peroxide by the mitochondrial 2-oxoadipate dehydrogenase complex. Free. Radic. Biol. Med. 2016, 91, 247-255. [CrossRef]

63. Nemeria, N.S.; Gerfen, G.; Guevara, E.; Reddy Nareddy, P.; Szostak, M.; Jordan, F. The human Krebs cycle 2-oxoglutarate dehydrogenase complex creates an additional source of superoxide/hydrogen peroxide from 2-oxoadipate as alternative substrate. Free Radic. Biol. Med. 2017, 108, 644-654. [CrossRef] [PubMed]

64. Jordan, F.; Nemeria, N.; Gerfen, G. Human 2-Oxoglutarate Dehydrogenase and 2-Oxoadipate Dehydrogenase Both Generate Superoxide $/ \mathrm{H}_{2} \mathrm{O}_{2}$ in a Side Reaction and Each Could Contribute to Oxidative Stress in Mitochondria. Neurochem. Res. 2019, 44, 2325-2335. [CrossRef] [PubMed] 
65. Frank, R.A.W.; Kay, C.W.M.; Hirst, J.; Luisi, B.F. Off-Pathway, Oxygen-Dependent Thiamine Radical in the Krebs Cycle. J. Am. Chem. Soc. 2008, 130, 1662-1668. [CrossRef]

66. Frank, R.A.; Price, A.J.; Northrop, F.D.; Perham, R.N.; Luisi, B.F. Crystal Structure of the E1 Component of the Escherichia coli 2-Oxoglutarate Dehydrogenase Multienzyme Complex. J. Mol. Biol. 2007, 368, 639-651. [CrossRef]

67. Wagner, T.; Bellinzoni, M.; Wehenkel, A.; O'Hare, H.M.; Alzari, P.M. Functional plasticity and allosteric regulation of $\alpha-$ ketoglutarate decarboxylase in central mycobacterium metabolism. Chem. Biol. 2011, 18, 1011-1020. [CrossRef]

68. Wagner, T.; Barilone, N.; Alzari, P.M.; Bellinzoni, M. A dual conformation of the post-decarboxylation intermediate is associated with distinct enzyme states in mycobacterial KGD ( $\alpha$-ketoglutarate decarboxylase). Biochem. J. 2014, 457, 425-434. [CrossRef]

69. Wagner, T.; Boyko, A.; Alzari, P.M.; Bunik, V.I.; Bellinzoni, M. Conformational transitions in the active site of mycobacterial 2-oxoglutarate dehydrogenase upon binding phosphonate analogues of 2-oxoglutarate: From a Michaelis-like complex to ThDP adducts. J. Struct. Biol. 2019, 208, 182-190. [CrossRef]

70. Zhou, J.; Yang, L.; Ozohanics, O.; Zhang, X.; Wang, J.; Ambrus, A.; Arjunan, P.; Brukh, R.; Nemeria, N.S.; Furey, W.; et al. A multipronged approach unravels unprecedented protein-protein interactions in the human 2-oxoglutarate dehydrogenase multienzyme complex. J. Biol. Chem. 2018, 293, 19213-19227. [CrossRef]

71. Mande, S.S.; Sarfaty, S.; Allen, M.D.; Perham, R.N.; Hol, W.G. Protein-protein interactions in the pyruvate dehydro-genase multienzyme complex: Dihydrolipoamide dehydrogenase complexed with the binding domain of dihydrolipoamide acetyltransferase. Structure 1996, 4, 277-286. [CrossRef]

72. Heublein, M.; Burguillos, M.A.; Vögtle, F.N.; Teixeira, P.F.; Imhof, A.; Meisinger, C.; Ott, M. The novel component Kgd4 recruits the E3 subunit to the mitochondrial $\alpha$-ketoglutarate dehydrogenase. Mol. Biol. Cell 2014, 25, 3342-3349. [CrossRef] [PubMed]

73. Leandro, J.; Dodatko, T.; Aten, J.; Nemeria, N.S.; Zhang, X.; Jordan, F.; Hendrickson, R.C.; Sanchez, R.; Yu, C.; DeVita, R.J.; et al. DHTKD1 and OGDH display substrate overlap in cultured cells and form a hybrid 2-oxo acid dehydrogenase complex in vivo. Hum. Mol. Genet. 2020, 29, 1168-1179. [CrossRef]

74. Harris, R.A.; Bowker-Kinley, M.M.; Wu, P.; Jeng, J.; Popov, K.M. Dihydrolipoamide Dehydrogenase-binding Protein of the Human Pyruvate Dehydrogenase Complex: DNA-derived amino acid sequence, expression, and reconstitution of the pyruvate dehydrogenase complex. J. Biol. Chem. 1997, 272, 19746-19751. [CrossRef] [PubMed]

75. Hiromasa, Y.; Fujisawa, T.; Aso, Y.; Roche, T.E. Organization of the Cores of the Mammalian Pyruvate Dehydrogenase Complex Formed by E2 and E2 Plus the E3-binding Protein and Their Capacities to Bind the E1 and E3 Components. J. Biol. Chem. 2004, 279, 6921-6933. [CrossRef] [PubMed]

76. Brautigam, C.A.; Wynn, R.M.; Chuang, J.L.; Chuang, D.T. Subunit and Catalytic Component Stoichiometries of an in Vitro Reconstituted Human Pyruvate Dehydrogenase Complex. J. Biol. Chem. 2009, 284, 13086-13098. [CrossRef]

77. Vijayakrishnan, S.; Kelly, S.M.; Gilbert, R.J.; Callow, P.; Bhella, D.; Forsyth, T.; Lindsay, J.G.; Byron, O. Solution structure and characterisation of the human pyruvate dehydrogenase complex core assembly. J. Mol. Biol. 2010, 399, 71-93. [CrossRef] [PubMed]

78. Vijayakrishnan, S.; Callow, P.; Nutley, M.A.; McGow, D.P.; Gilbert, D.; Kropholler, P.; Cooper, A.; Byron, O.; Lindsay, J.G. Variation in the organization and subunit composition of the mammalian pyruvate dehydrogenase complex E2/E3BP core as-sembly. Bioch. J. 2011, 437, 565-574. [CrossRef]

79. Jiang, J.; Baiesc, F.L.; Hiromasa, Y.; Yu, X.; Hui, W.H.; Dai, X.; Roche, T.E.; Zhou, Z.H. Atomic Structure of the E2 Inner Core of Human Pyruvate Dehydrogenase Complex. Biochemistry 2018, 57, 2325-2334. [CrossRef]

80. Prajapati, S.; Haselbach, D.; Wittig, S.; Patel, M.S.; Chari, A.; Schmidt, C.; Stark, H.; Tittmann, K. Structural and Functional Analyses of the Human PDH Complex Suggest a "Division-of-Labor" Mechanism by Local E1 and E3 Clusters. Structure 2019, 27, 1124-1136.e4. [CrossRef]

81. Steginsky, C.A.; Gruys, K.J.; Frey, P.A. Alpha-Ketoglutarate dehydrogenase complex of Escherichia coli. A hybrid complex containing pyruvate dehydrogenase subunits from pyruvate dehydrogenase complex. J. Biol. Chem. 1985, 260, 13690-13693. [CrossRef]

82. Niebisch, A.; Kabus, A.; Schultz, C.; Weil, B.; Bott, M. Corynebacterial protein kinase G controls 2-oxoglutarate de-hydrogenase activity via the phosphorylation status of the OdhI protein. J. Biol. Chem. 2006, 281, 12300-12307. [CrossRef]

83. Hoffelder, M.; Raasch, K.; Van Ooyen, J.; Eggeling, L. The E2 Domain of OdhA of Corynebacterium glutamicum Has Succinyltransferase Activity Dependent on Lipoyl Residues of the Acetyltransferase AceF. J. Bacteriol. 2010, 192, 5203-5211. [CrossRef]

84. Tian, J.; Bryk, R.; Shi, S.; Erdjument-Bromage, H.; Tempst, P.; Nathan, C. Mycobacterium tuberculosis appears to lack $\alpha-$ ketoglutarate dehydrogenase and encodes pyruvate dehydrogenase in widely separated genes. Mol. Microbiol. 2005, 57, 859-868. [CrossRef] [PubMed]

85. Zhang, X.; Nemeria, N.S.; Leandro, J.; Houten, S.; Lazarus, M.B.; Gerfen, G.J.; Ozohanics, O.; Ambrus, A.; Nagy, B.; Brukh, R.; et al. Structure-function analyses of the G729R 2-oxoadipate dehydrogenase genetic variant associated with a disorder of 1-lysine metabolism. J. Biol. Chem. 2020, 295, 8078-8095. [CrossRef] [PubMed]

86. Luan, C.-J.; Guo, W.; Chen, L.; Wei, X.-W.; He, Y.; Chen, Y.; Dang, S.-Y.; Prior, R.; Li, X.; Kuang, Y.; et al. CMT2Q-causing mutation in the Dhtkd1 gene lead to sensory defects, mitochondrial accumulation and altered metabolism in a knock-in mouse model. Acta Neuropathol. Commun. 2020, 8, 1-13. [CrossRef] [PubMed]

87. Lindqvist, Y.; Schneider, G.; Ermler, U.; Sundström, M. Three-dimensional structure of transketolase, a thiamine diphosphate dependent enzyme, at 2.5 A resolution. EMBO J. 1992, 11, 2373-2379. [CrossRef] [PubMed] 
88. Muller, Y.A.; Lindqvist, Y.; Furey, W.; Schulz, G.E.; Jordan, F.; Schneider, G. A thiamin diphosphate binding fold revealed by comparison of the crystal structures of transketolase, pyruvate oxidase and pyruvate decarboxylase. Structure 1993, 1,95-103. [CrossRef]

89. Aevarsson, A.; Seger, K.; Turley, S.; Sokatch, J.R.; Hol, W.G. Crystal structure of 2-oxoisovalerate and dehydrogenase and the architecture of 2-oxo acid dehydrogenase multienzyme complexes. Nat. Genet. 1999, 6, 785-792. [CrossRef]

90. Frank, R.A.; Pratap, J.V.; Pei, X.Y.; Perham, R.N.; Luisi, B.F. The Molecular Origins of Specificity in the Assembly of a Multienzyme Complex. Structure 2005, 13, 1119-1130. [CrossRef]

91. Park, Y.-H.; Wei, W.; Zhou, L.; Nemeria, N.; Jordan, F. Amino-terminal residues 1-45 of the Escherichia coli pyruvate dehydrogenase complex E1 subunit interact with the E2 subunit and are required for activity of the complex but not for reductive acetylation of the E2 subunit. Biochemistry 2004, 43, 14037-14046. [CrossRef] [PubMed]

92. Song, J.; Park, Y.-H.; Nemeria, N.S.; Kale, S.; Kakalis, L.; Jordan, F. Nuclear Magnetic Resonance Evidence for the Role of the Flexible Regions of the E1 Component of the Pyruvate Dehydrogenase Complex from Gram-negative Bacteria. J. Biol. Chem. 2010, 285, 4680-4694. [CrossRef] [PubMed]

93. Hengeveld, A.F.; De Kok, A. Identification of the E2-binding residues in the N-terminal domain of E1 of a prokaryotic pyruvate dehydrogenase complex. FEBS Lett. 2002, 522, 173-176. [CrossRef]

94. Zhang, X. Study of the Structure and Interactions of Components in the 2-Oxoglutarate Dehydrogenase and 2-Oxoadipate Dehydrogenase Complexes. Ph.D. Thesis, Rutgers University, Newark, NJ, USA, 2021.

95. Orbán-Németh, Z.; Beveridge, R.; Hollenstein, D.M.; Rampler, E.; Stranzl, T.; Hudecz, O.; Doblmann, J.; Schlögelhofer, P.; Mechtler, K. Structural prediction of protein models using distance restraints derived from cross-linking mass spectrometry data. Nat. Protoc. 2018, 13, 478-494. [CrossRef] [PubMed]

96. Fux, A.; Korotkov, V.S.; Schneider, M.; Antes, I.; Sieber, S.A. Chemical Cross-Linking Enables Drafting ClpXP Proximity Maps and Taking Snapshots of In Situ Interaction Networks. Cell Chem. Biol. 2019, 26, 48-59. [CrossRef]

97. Roy, A.; Kucukural, A.; Zhang, Y. I-TAASSER: A unified platform for automated protein structure and function pre-diction. Nat. Protoc. 2010, 5, 725-738. [CrossRef]

98. Kosinski, J.; von Appen, A.; Ori, A.; Karius, K.; Muller, C.W.; Beck, M. Xlink analyzer: Software for analysis and visualization of cross-linking data in the context of three-dimensional structures. J. Struct. Biol. 2015, 189, 177-183. [CrossRef] [PubMed]

99. Pettersen, E.F.; Goddard, T.D.; Huang, C.C.; Couch, G.S.; Greenblatt, D.M.; Meng, E.C.; Ferrin, T.E. UCSF chimera-a visualization system for exploratory research and analysis. J. Comput. Chem. 2004, 25, 1605-1612. [CrossRef]

100. Kahraman, A.; Herzog, F.; Leitner, A.; Rosenberger, G.; Aebersold, R.; Malmström, L. Cross-Link Guided Molecular Modeling with ROSETTA. PLoS ONE 2013, 8, e73411. [CrossRef]

101. Yan, Y.; Tao, H.; He, J.; Huang, S.-Y. The HDOCK server for integrated protein-protein docking. Nat. Protoc. 2020, 15, 1829-1852. [CrossRef]

102. Götze, M.; Pettelkau, J.; Fritzsche, R.; Ihling, C.H.; Schäfer, M.; Sinz, A. Automated Assignment of MS/MS Cleavable Cross- Links in Protein 3D-Structure Analysis. J. Am. Soc. Mass Spectrom. 2014, 26, 83-97. [CrossRef]

103. Grimm, M.; Zimniak, T.; Kahraman, A.; Herzog, F. xVis: A web server for the schematic visualization and interpretation of crosslink-derived spatial restraints. Nucleic Acids Res. 2015, 43, W362-W369. [CrossRef]

104. Zhang, X.; Nemeria, N.S.; Jordan, F. Understanding of the 2-oxoglutarate dehydrogenase and 2-oxoadipate dehydrogenase assembly with the E2o core relevant to a hybrid complex formation. bioRxiv 2021. [CrossRef]

105. Wagenknecht, T.; Grassucci, R.; Schaak, D. Cryoelectron microscopy of frozen-hydrated $\alpha$-ketoacid dehydrogenase complexes from Escherichia Coli. J. Biol. Chem. 1990, 265, 22402-22408. [CrossRef]

106. Murphy, G.E.; Jensen, G.J. ; Jensen, G.J. Electron cryotomography of the E. coli pyruvate and 2-oxoglutarate dehydrogenase complexes. Structure 2005, 13, 1765-1773. [CrossRef]

107. Milne, J.L.S.; Wu, X.; Borgnia, M.J.; Lengyel, J.S.; Brooks, B.R.; Shi, D.; Perham, R.N.; Subramaniam, S. Molecular structure of a 9-MDa icosahedral pyruvate dehydrogenase subcomplex containing the E2 and E3 enzymes using cryoelectron microscopy. $J$. Biol. Chem. 2005, 281, 4364-4370. [CrossRef] [PubMed]

108. Stoops, J.K.; Baker, T.S.; Schroeter, J.P.; Kolodziej, S.J.; Niu, X.-D.; Reed, L.J. Three dimensional structure of the truncated core of the Saccharomyces cerevisiae pyruvate dehydrogenase complex determined from negative stain and cryoelectron microscopy images. J. Biol. Chem. 1992, 267, 24769-24775. [CrossRef]

109. Stoops, J.K.; Cheng, R.H.; Yazdi, M.A.; Maeng, C.-Y.; Schroeter, J.P.; Klueppelberg, U.; Kolodziej, S.J.; Baker, T.S.; Reed, L.J. On the unique structural organization of the Saccaromyces cerevisiae pyruvate dehydrogenase complex. J. Biol. Chem. 1997, 272, 5757-5764. [CrossRef] [PubMed]

110. Gu, Y.; Zhou, Z.H.; McCarthy, D.B.; Reed, L.J.; Stoops, J.K. 3D electron microscopy reveals the variable deposition and protein dynamics of the peripheral pyruvate dehydrogenase component about the core. Proc. Natl. Acad. Sci. USA 2003, 100, 7015-7702. [CrossRef]

111. Wagenknecht, T.; Grassucci, R.; Radke, G.A.; Roche, T. Cryoelectron microscopy of mammalian pyruvate dehydrogenase com plex. J. Biol. Chem. 1991, 266, 24650-24656. [CrossRef] 
112. Zhou, Z.H.; McCarthy, D.B.; O'Connor, C.M.; Reed, L.J.; Stoops, J.K. The remarkable structural and functional organization of the eukaryotic pyruvate dehydrogenase complexes. Proc. Nat. Acad. Sci. USA 2001, 98, 14802-14807. [CrossRef] [PubMed]

113. Reed, L.J.; Hackert, M.L. Structure-function relationships in dihydrolipoamide acyltransferases. J. Biol. Chem. 1990, 265, 8971-8974. [CrossRef] 